\title{
Electron-density descriptors as predictors in quantitative structure-activity/property relationships and drug design
}

The use of electron density-based molecular descriptors in drug research, particularly in quantitative structureactivity relationships/quantitative structure-property relationships studies, is reviewed. The exposition starts by a discussion of molecular similarity and transferability in terms of the underlying electron density, which leads to a qualitative introduction to the quantum theory of atoms in molecules (QTAIM). The starting point of QTAIM is the topological analysis of the molecular electron-density distributions to extract atomic and bond properties that characterize every atom and bond in the molecule. These atomic and bond properties have considerable potential as bases for the construction of robust quantitative structure-activity/property relationships models as shown by selected examples in this review. QTAIM is applicable to the electron density calculated from quantum-chemical calculations and/or that obtained from ultra-high resolution $\mathrm{x}$-ray diffraction experiments followed by nonspherical refinement. Atomic and bond properties are introduced followed by examples of application of each of these two families of descriptors. The review ends with a study whereby the molecular electrostatic potential, uniquely determined by the density, is used in conjunction with atomic properties to elucidate the reasons for the biological similarity of bioisosteres.

Quantitative structure-activity (or property) studies are often constructed from $a d$ hoc sets of molecular descriptors with the goal of obtaining good predictive statistics. Not infrequently, the descriptors are chosen solely for the improvement they bring to the statistics with little or no emphasis on their physical meaning in relation to the predicted activity or property. While this strategy is undeniably useful for the development of new drugs, it is argued that better modeling can be achieved on the basis of well-defined physical descriptors. The recommended descriptors have simple physicochemical interpretations and, in addition to removing an important element of uncertainty in the modeling, provide valuable physicochemical insight. These descriptors are derived from the topological analysis of the electron density and from the associated molecular electrostatic potential (MEP), both experimentally accessible scalar fields fundamentally related to all molecular ground-state properties. This review demonstrates the validity of this standpoint by showing how few electrondensity and electrostatic-potential descriptors can be used to construct physically meaningful predictive quantitative structure-activity relationships/quantitative structure-property relationships (QSAR/QSPR) models.
- Molecular similarity

In studies of QSAR or QSPR, empirical relations are constructed with the goal of predicting the activity (or properties) of compounds given known properties of a series of congeners. In typical cases, the compounds chosen in the training set share a degree of structural similarity, a well-known example being substituted benzoic acids and the Hammett constant.

Chemists have an intuitive appreciation of the degree of similarity of compounds, an appreciation that guides the selection of the members of the molecular set used in the construction of QSAR/QSPR models. It must be noted, however, that there are QSAR/QSPR models that are constructed on the basis of physicochemical properties (e.g., $\log \mathrm{P}$ ) rather than structural descriptors (e.g., geometrical parameters) in which case the compound libraries can be far more diverse and do not necessarily take structural similarity into consideration.

The concept of molecular similarity permeates much of medicinal chemistry, to one degree or another, especially in the conceptualization of new drug molecules [1-6]. The observations outlined previously raise the question of whether molecular similarity can be quantified

Chérif F Matta ${ }^{\dagger 1,2}$ \&
Alya A Arabi,
'Department of Chemistry and
Physics, Mount Saint Vincent
University, Halifax, Nova Scotia,
Canada B3M 2J6
2Department of Chemistry, Dalhousie
University, Halifax, Nova Scotia,
Canada B3H 4J3
†Author for correspondence:
Tel.: + 9024576142
Fax: +19024576134
E-mail: cherif.matta@msvu.ca

FUTURE
SCIENCE 
objectively. This concept can be applied in the context of whole molecules or, alternatively, to particular common molecular fragment(s), such as pharmacophores or toxicophores.

Similarity is a relational concept that can only be formulated with respect to a given quality or property, for example molecular geometry, molecular weight and empirical formula. There is no shortage of molecular similarity studies applied to different properties, be it at the level of amino acid sequences [7-9], chemical 2D graphs [10], geometrical superimpositions [11], superimpositions of molecular electron densities [1-4], or comparisons of the MEP [12-17]. But which approach captures molecular similarity to a maximal extent, and why? Is the answer to this question a function of the problem or is it universal? It is argued in this review that, in principle, the answer is unique: the molecular electron density $\rho(\mathbf{r})$ captures and determines all the properties of the molecule. In practice, however, the functional relationship between the density and the various properties is often unknown or known only approximately. The properties derived from the topology of the electron density hold, thus, much promise since their choice eliminates an important source of uncertainty in the modeling. The properties derived from the electron density can yield strong correlations that can be used in the prediction of the properties of unknown compounds. At the same time, these density-derived properties are shown to provide valuable physicochemical and mechanistic insight.

$$
\rho(\mathbf{r}) \rightarrow\left\{\begin{array}{l}
v[\rho(\mathbf{r})] \\
N[\rho(\mathbf{r})]
\end{array}\right\} \rightarrow \hat{H}[\rho(\mathbf{r})] \rightarrow \Psi[\rho(\mathbf{r})] \rightarrow O[\rho(\mathbf{r})]
$$

The first HK theorem implies that if the electron densities of two systems are similar, then the similarity of all other properties necessarily follows. There is, thus, a strong theoretical justification for choosing the electron density as the basic object of study in molecular similarity, in the construction of QSAR, in studies of molecular complementarity and drug-receptor interaction. The HK theorem, in its original formulation, applies to closed (full) quantum systems the electron density of which extends to infinity in the usual 3D space. More recently, Riess and Münch's extended the HK theorem to arbitrarily small finite enclosed subsystems [19]. They have shown that a bounded region of electron density inside a molecule, that is to say, an open quantum system capable of exchanging matter and energy with its surrounding, is uniquely mapped to all ground-state properties (including the electron density) of the full system [19]. (See [20] for a discussion of the implications of this important extension of the HK theorem on the transferability of atoms in molecules and [21] for its implications on Carbó's similarity measure).

Given that the electron density is the basic object in a molecular similarity study, the question of how to compare the electron densities of two molecules is yet to be settled definitively. A widely accepted definition to quantify the similarity of the electron density distributions of two molecules is the Carbó molecular similarity index, defined as $[1,4]$ :

$$
\frac{C_{A B}(\hat{O} ; \Theta)=}{\sqrt{\iint \rho_{A}\left(\mathbf{r}_{1}\right) \hat{O}\left(\mathbf{r}_{1}, \mathbf{r}_{2}\right) \rho_{A}\left(\mathbf{r}_{2}\right) d \mathbf{r}_{1} d \mathbf{r}_{2} \iint \rho_{B}\left(\mathbf{r}_{1}\right) \hat{O}\left(\mathbf{r}_{1}, \mathbf{r}_{2}\right) \rho_{B}\left(\mathbf{r}_{2}\right) d \mathbf{r}_{1} d \mathbf{r}_{2}}}
$$

EQUATION 2

A fundamental tenet of modern density-functional theory (DFT) is the well-known (first) Hohenberg-Kohn (HK) theorem [18]. This theorem states that there exists a unique functional mapping between the ground-state electron density, $\rho(\mathbf{r})$, and the external potential, $v[\rho(\mathbf{r})]$, and the number of electrons, $N[\rho(\mathbf{r})]$. Since $v[\rho(\mathbf{r})]$ and $N[\rho(\mathbf{r})]$ completely specify the hamiltonian, therefore, the density determines the wavefunction of the system $\Psi[\rho(\mathbf{r})]$. Quantum mechanics teaches us that the wavefunction contains all the information that can be known about the system, and thus, there exists a unique mapping between the electron density and all the properties of the system $O[\rho(\mathbf{r})]$. Symbolically: where $\rho_{A}$ and $\rho_{B}$ are the electron density distributions of molecules $A$ and $B$, respectively, and the integral is over all $3 \mathrm{D}$ space. Different operators $\hat{O}\left(\mathbf{r}_{1}, \mathbf{r}_{2}\right)$ in Equation 2 result in different definitions of the similarity index. Two commonly used operators are [4]:

- $\hat{O}\left(\mathbf{r}_{1}, \mathbf{r}_{2}\right)=\delta\left(\mathbf{r}_{1}-\mathbf{r}_{2}\right)$, a Dirac $\delta$ that reduces the numerator to $\int \rho_{A}(\mathbf{r}) \rho_{B}(\mathbf{r}) d \mathbf{r}$ and yielding the well-known Carbó overlap similarity index in its most recognizable form:

$$
C_{A B}=\frac{\int \rho_{\mathrm{A}} \rho_{\mathrm{B}} \mathrm{dv}}{\sqrt{\int \rho_{\mathrm{A}}^{2} \mathrm{dv} \int \rho_{\mathrm{B}}^{2} \mathrm{dv}}}
$$

EQUATION 3 
- $\hat{O}\left(\mathbf{r}_{1}, \mathbf{r}_{2}\right)=\left|\mathbf{r}_{1}-\mathbf{r}_{2}\right|^{-1}$, the Coulomb operator in which case the numerator becomes $\iint \rho_{A}\left(\mathbf{r}_{1}\right)\left|\mathbf{r}_{1}-\mathbf{r}_{2}\right|^{-1} \rho_{B}\left(\mathbf{r}_{2}\right) d \mathbf{r}_{1} d \mathbf{r}_{2}$ yielding a Coulomb similarity index.

The denominator in Equation $\mathbf{2}$ has the role of a normalization factor (since when $\rho_{A}=\rho_{B}$ the denominator causes $C_{A B}$ to become unity). Thus, Carbó-type indexes defined in Equation 2 are bound by the maximal value of 1 , which indicates 'identity' and can approach (but never reach) zero, that is, $0<C_{A B} \leq 1$. Finally, the parameter $\Theta$ represents the alignment orientation of the two molecules being compared. The same form of the index given in Equation 2 can be used to compare MEPs after replacing molecular electron densities by MEPs $V(\mathbf{r})$.

The calculation of Carbó's index, $C_{A B}$, brings to the fore the problem of alignment: how to align the two densities $\rho_{A}$ and $\rho_{B}$ (i.e., what is the optimal $\Theta$ ) before integrating their product? An approach to this alignment problem is based on maximizing the index $[4,22,23]$, which is equivalent to maximizing the integral $\int \rho_{A} \rho_{B} d v$ as indicated in Equation 2. The maximization of the numerator of EQUATION $\mathbf{2}$ is computationally prohibitive since the integral has to be evaluated for each small change of the relative orientations $\Theta$ of $A$ and $B$. Thus, the alignment problem is a drawback of Carbó's similarity index.

Another limitation of the $C_{A B}$ index is the dominance of the core densities in determining its magnitude. The maxima of density at and near the nuclei exaggerate the mismatches (dissimilarity) of densities that, with the exception of slight variations in bond lengths, are very similar. In other words, the nuclear maxima render the comparison more sensitive to chemically irrelevant regions near the atomic nuclei at the expense of the more diffuse but more chemically relevant regions of chemical bonding and lone pairs.

This article reviews some of the methods that were developed as alternatives to Carbó's index on the basis of a theory that takes the electron density as its starting point. This theory is termed the quantum theory of atoms in molecules (QTAIM) [24-29].

- Transferability of atoms \& groups of atoms between molecules

Closely related to the concept of similarity is that of transferability, that is, the retention of approximately invariant properties of an atom or a group of atoms embedded in different molecular environments. Perfect transferability is (trivially) only achievable at the limit of perfect similarity, namely, identity. Any change in the external potential, including minor conformational changes, precludes perfect transferability.

The nonexistence of perfect transferability is a manifestation of the first HK theorem (Equation I). In an extension of the HK theorem, Reiss and Münch [19], Bader and Becker [20], and Mezey [21] have shown that the electron density of an arbitrarily small region is mapped bijectively to the full density. Therefore, no two atoms or functional groups can be identical in different molecules. For example, if two molecules of a gigantic protein differ in just the conformation of a single amino acid residue side chain, then no two atoms in these two molecules are identical. However, and as pointed out by Bader and Baker [20], the HK theorem does not impose any limit on how similar (transferable) two atoms in different molecules can be. In practice, perfect similarity can (and is) often achieved within chemical precision ( $\leq 1 \mathrm{kcal} / \mathrm{mol}$ ). In other words, the impossibility of total similarity or perfect transferability does not preclude near-perfect practical transferability. It is in virtue of this practical transferability that atoms and functional groups retain experimentally recognizable properties that are only perturbed by the electronic environment.

The related concepts of similarity and transferability are pervasive in medicinal chemistry, particularly in the in silico phase of the design of novel leads and in the fine-tuning of the pharmacokinetics and pharmacodynamics of known scaffolds.

\section{Analysis of the electron density: the QTAIM}

The QTAIM is often used to analyze and compare electron-density distributions, whether obtained from theoretical calculations [26,27] or from multipolar refinement of high-resolution $\mathrm{x}$-ray diffraction data [29-31]. It is beyond the scope of this article to discuss this theory, which has been amply reviewed in the literature [24-31]. We only remind the reader with a few of the most basic concepts of this theory that are necessary to understand the remainder of this article.

At the present stage of development, the use of $x$-ray electron densities in routine QSAR/ QSPR studies is impractical since one has to first be able to obtain good-quality crystals followed by a nontrivial experimental and refinement procedure for each molecule included in 
the training set. This limitation does not apply for electron densities calculated from quantum mechanics, especially with the considerable and fast evolution of computer hardware and software. The quantum mechanical calculation of the electron density for fairly large molecules has become fast, routine, and standardized, thanks to software such as Gaussian [32] and GAMESS [33], for example.

Molecular electron densities are characterized by dominant local 3D maxima where the nuclei are located. Figure I represents a relief map of the electron density in the plane of the phenolic ring in the morphine molecule. Remarkably, the pattern of chemical bonds linking the atoms, that is the chemical structure, can already be seen emerging from this representation of $\rho(\mathbf{r})$. The relief map reveals a ridge of density, with a point having the appearance of a saddle point, connecting any two nuclei of a pair of bonded atoms. These saddle points are termed bond critical points (BCPs). The BCP is special point in space where the electron density is minimal along the bond path (the path that links two atoms) but maximal in any other direction. The gradient path originating at the BCP and terminating at the nuclei is a line of locally maximum density termed the bond path [34,35]. There exists such a bond path between every pair of chemically bonded atoms.

An isocontour plot of the electron density drawn on the molecular plane of the phenolic ring of morphine is displayed in Figure 2.

In this figure, the lines linking the nuclei are the bond paths. The atoms are delimited by welldefined boundaries that have the appearance of lines enclosing any given mononuclear region (an atom-in-a-molecule). the empirically known chemical structure is mirrored faithfully by the corresponding molecular graph obtained directly from the electron density as a set of bond paths. It is particularly fascinating that weak bonds, such as hydrogen bonds, are easily identified, without the usual ambiguities, from the topology of the electron density.

The intersection of the inter-atomic surfaces that partition space into nonoverlapping mononuclear regions is represented in Figure $\mathbf{2}$ as lines bounding the atoms. These interatomic surfaces are never crossed by the gradient vectors and, thus, satisfy the following condition locally:

$\nabla \rho(\mathbf{r}) \cdot \mathbf{n}(\mathbf{r})=0$

for all $\mathbf{r}$ belonging to the surface $S(\Omega)$

\section{EOUATION 4}

where $\mathbf{r}$ is the position vector and $\mathbf{n}(\mathbf{r})$ the unit vector normal to the surface $S(\Omega)$.

\section{- QTAIM atomic properties}

The partitioning of a molecule into nonoverlapping atoms entails the partitioning of molecular properties that are expressible as real-space densities into additive transferable atomic contributions. This is achieved by integrating the corresponding density over a given bounded atomic volume. Examples of such properties include atomic charges (the zero ${ }^{\text {th }}$-order atomic electrostatic multipole) and higher order electrostatic multipoles, atomic energies, atomic volumes and polarizabilities.

Thus, for a system at electrostatic equilibrium, the expectation value of an operator averaged over all space can be written as a sum of the expectation values of this operator averaged over each individual atom $\Omega$ in the molecule or the crystal, that is:

$$
\begin{aligned}
& \langle\hat{O}\rangle_{\text {molecule }}=\sum_{i}^{\substack{\text { all atoms } \\
\text { in the } \\
\text { molecule }}}\left[N \iint_{\Omega_{i}}\left\{\int \frac{1}{2}[\Psi * \hat{O} \Psi+(\hat{O} \Psi) * \Psi] d \tau^{\prime}\right\} d \mathbf{r}\right] \\
& =\sum_{i}^{\substack{\text { all atoms } \\
\text { in the } \\
\text { molecule }}}\left[\int_{\Omega_{i}} \rho_{O} d \mathbf{r}\right]=\sum_{i}^{\substack{\text { all atoms } \\
\text { in the } \\
\text { molecule }}}\left\langle\hat{O}\left(\Omega_{i}\right)\right\rangle
\end{aligned}
$$

These lines are the intersections of the socalled zero-flux interatomic surfaces with the plane of the figure. The bond critical point lies at the intersection of the bond path with the corresponding zero-flux interatomic surface. The collection of bond paths recover the chemical structure inferred from empirical evidence as illustrated in Figure 3. As depicted in Figure 3,
Equation 5

where $\left\langle\hat{O}_{\text {molecule }}\right\rangle$ is the molecular average of a linear hermitian operator $\hat{O}$ and $\left\langle\hat{O}\left(\Omega_{i}\right)\right\rangle$ its atomic average. The last equality of EQuation 5 expresses the additivity of the so-defined atomic properties. Thus, for a physical property $O$ that can be expressed in terms of a real space density $\rho_{O}(\mathbf{r})$, the molecular value can be expressed as a sum 
of atomic contributions, each being the average of the corresponding operator over the atomic volume Vol. $(\Omega)$.

As an example, the atomic electrostatic monopole (or atomic charge), is given by the difference between the atomic population of atom $\Omega$, $N(\Omega)$ (obtained when $\hat{O}=\hat{1}$ ), and the charge of its nucleus $Z_{\Omega}$. The atomic dipole moment, is obtained by:

$$
\mu(\Omega)=-e \int_{\Omega} r \Omega \rho(\mathbf{r}) d \mathbf{r}
$$

Equation 6 where the origin is placed at the position of the nucleus of atom $\Omega$. The MEP has been shown to be accurately reproduced from QTAIM multipoles on a number of occasions [36-39].

\section{- QTAIM bond properties}

The concentration or depletion of electron density is revealed from the eigenvalues of the hessian matrix, which are the ordered collection of partial second derivatives of the electron density with respect to the Cartesian coordinates. The hessian can always be diagonalized, which is equivalent to performing a rotation of the coordinate system, but the trace (the sum of the diagonal elements) of the hessian remains invariant to such unitary transformations. The trace of the diagonalized hessian evaluated at the bond critical point is the sum of the three curvatures of the electron density at that point, also known as the laplacian of the density at the BCP:

$$
\nabla^{2} \rho_{\mathrm{BCP}}=\nabla \cdot \nabla \rho_{\mathrm{BCP}}=\frac{\frac{\partial^{2} \rho_{\mathrm{BCP}}}{\partial x^{2}}}{\lambda_{1}}+\underbrace{\frac{\partial^{2} \rho_{\mathrm{BCP}}}{\partial y^{2}}}_{\lambda_{2}}+\frac{\frac{\partial^{2} \rho_{\mathrm{BCP}}}{\partial z^{2}}}{\lambda_{3}}
$$

EQuATION 7

where $\lambda_{i}$ is the $i^{\text {th }}$ curvature.

Thus, at a BCP, the eigenvalues of the diagonalized terms of the Hessian matrix are $\lambda_{1}, \lambda_{2}$ and $\lambda_{3}$. Two (negative) eigenvalues are perpendicular to the bond path and to each other and the third (positive) eigenvalue is tangent to the bond path at the BCP. By convention, $\lambda_{1}$ and $\lambda_{2}$ denote the negative curvatures $\left(\lambda_{1}\right.$ being the negative curvature of largest magnitude) and $\lambda_{3}$ denotes the single positive curvature.

The sign and magnitude of $\nabla^{2} \rho_{\mathrm{BCP}}$ provides a convenient classification of bonding. A $\nabla^{2} \rho_{\mathrm{BCP}}<0$ is dominated by the negative eigenvalues $\left(\lambda_{1}\right.$ and $\left.\lambda_{2}\right)$ and diagnoses a shared bonding interaction. If the electron density is highly polarized, as in ionic or closed-shell bonding, then the positive eigenvalue $\left(\lambda_{3}\right)$ is dominant and the laplacian at the BCP is positive $\left(\nabla^{2} \rho_{\mathrm{BCP}}>0\right)$.
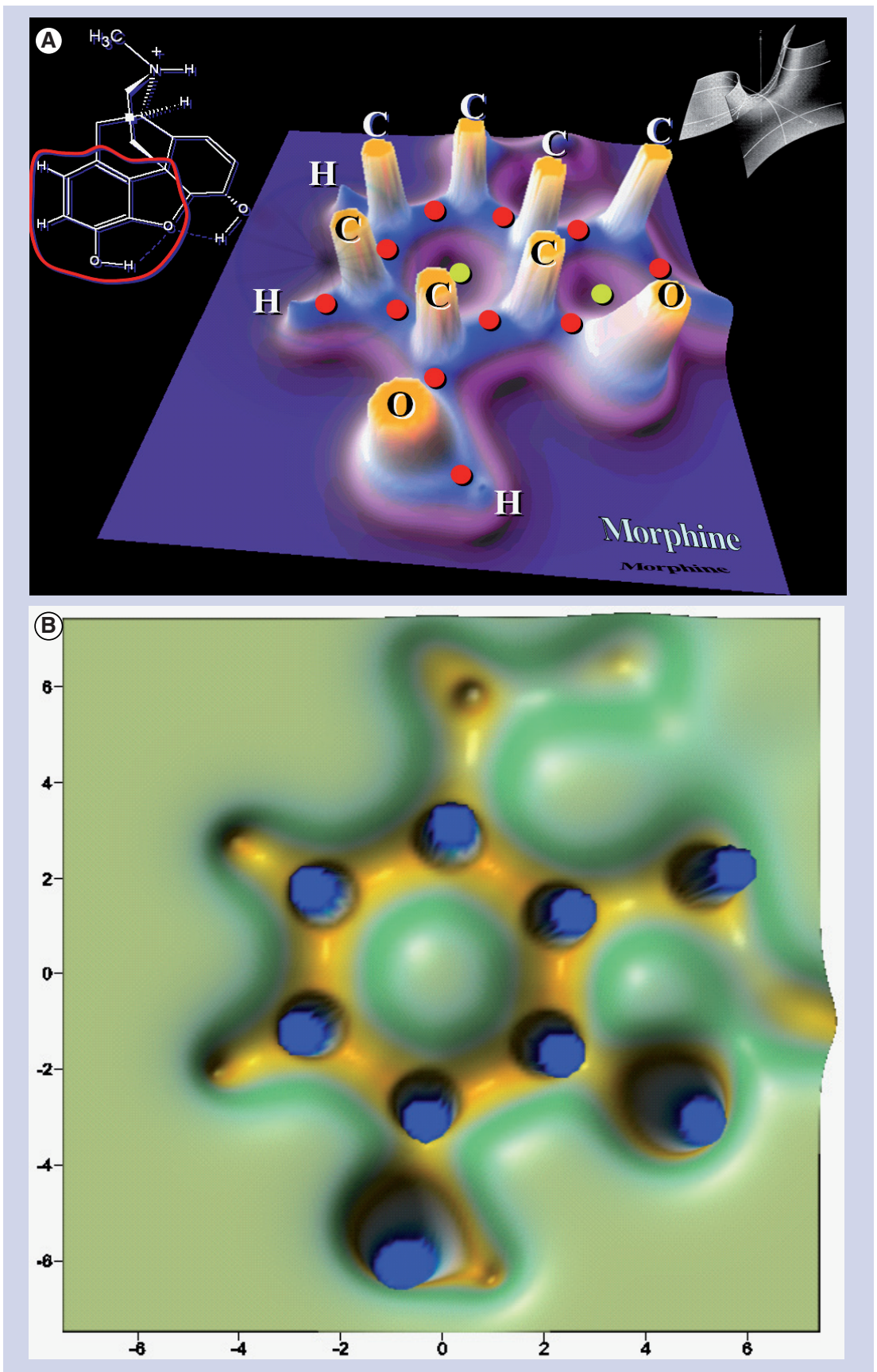

Figure 1. (A) Relief map of the electron density in the molecular plane of the phenolic ring of morphine; along with the chemical structure. The value of $\rho(\mathbf{r})$ is truncated to $1.0 \mathrm{au}$. Bond critical points are denoted by a red dot. Ring critical points are denoted by a yellow dot. (B) View of the same relief map in (A), where the ring is in the plane of the paper. The chemical structure of this portion of the morphine molecule emerges in complete accordance with the molecular structure representation linking bonded atoms by a 'stick'. The parts of the molecules to the right is not coplanar with the ring and, hence, is less well represented in the plane of the plots. The $x$ - and $y$-axes are labeled in atomic units of length ( 1 au of length $=1$ bohr $=\mathrm{a} 0$ ). 

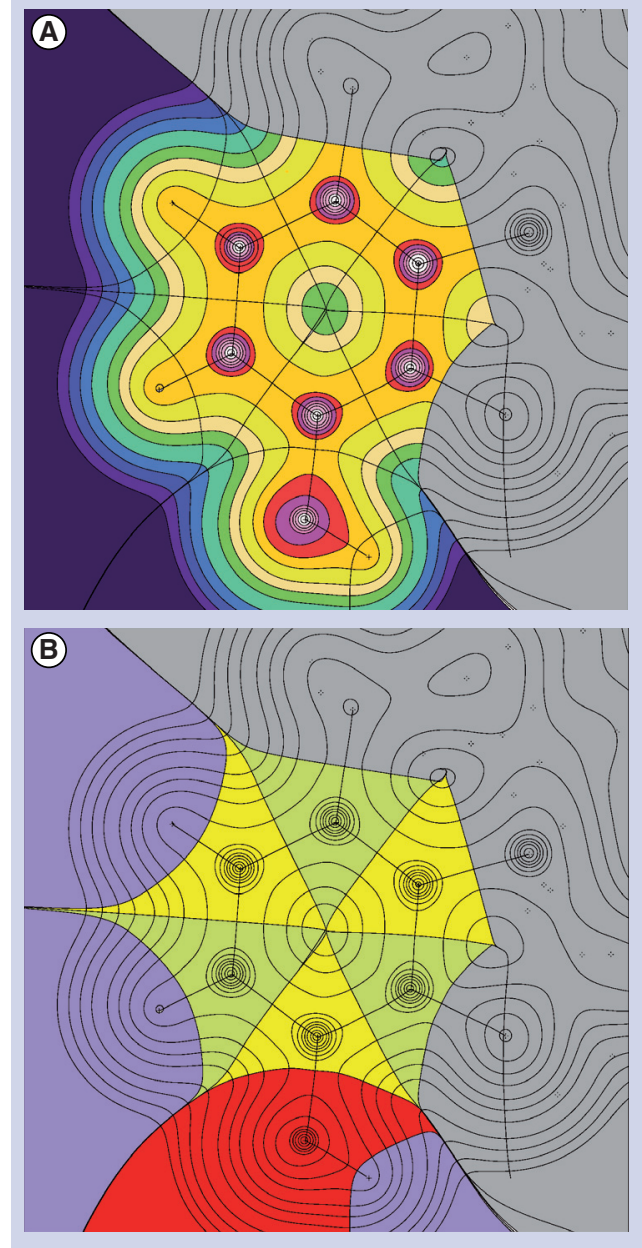

Figure 2. (A) Contour map of $\rho(\mathbf{r})$ in the plane of the phenolic ring of morphine corresponding to the relief map in Figure I. The outermost contour has the isodensity value of $0.001 \mathrm{au}$ followed by $2 \times 10^{n}, 4 \times 10^{n}$, and $8 \times 10^{n}$ au with $n$ starting at -3 and increasing in steps of unity. The regions between contours are colored to help guide the eye. Each contiguous line indicates a constant value of the electron density. The lines connecting the nuclei are the bond paths, and the lines delimiting each atom are the intersection of the interatomic zero-flux surfaces with the plane of the figure. The intersection of a bond path with an associated interatomic surface occurs at the bond critical point, where $\nabla \rho(\mathbf{r})=0$. (B) Same contour map as in (A) but color coded by element: $\mathrm{O}=$ red, $C=$ yellow or green (to distinguish adjacent $C$ atoms), $\mathrm{H}=$ violet).

The eigenvalues can also be used to calculate a bond 'ellipticity', $\varepsilon$, as a measure of the departure from cylindrical symmetry as in $\pi$-bonding for example. The bond ellipticity is defined as:

$$
\varepsilon=\frac{\lambda_{1}}{\lambda_{2}}-1
$$

From the definition in Equation 8, it is clear that $\varepsilon$ is always positive and it equals zero only for a perfectly cylindrical bond. The departure from 0 measures the preferential accumulation of charge in a given plane.

Energy densities require information contained in the one-electron density matrix (and not just the density [i.e., its diagonal elements]). The energy densities (potential, kinetic, and total) evaluated at the BCP are used to summarize the mechanics of a bonding interaction.

The average effective potential field experienced by a single electron at point $\mathbf{r}$, the virial field denoted by $\nu(\mathbf{r})$ is always negative and yields the total potential energy of the molecule when integrated over all space. It is locally related to the (gradient) electronic kinetic energy density $G(\mathbf{r})$ and the laplacian $[40,41]$ :

$$
\left[\frac{\hbar^{2}}{4 m}\right] \nabla^{2} \rho(\mathbf{r})=2 G(\mathbf{r})+v(\mathbf{r})
$$

EQUATION 9

in which

$$
G(\mathbf{r})=\frac{\hbar^{2}}{2 m} N \int d \tau^{\prime} \nabla \Psi^{*} \cdot \nabla \Psi
$$

EQUATION 10

where $\psi$ is an antisymmetric many-electron wavefunction. Equation 9 is known as the local virial theorem and was first derived by Bader [26].

Since $G(\mathbf{r})>0$ and $\nu(\mathbf{r})<0$ everywhere in space (including BCPs), the local virial theorem implies that bonding interactions for which $\nabla^{2} \rho_{\mathrm{BCP}}<0$ are dominated by a local lowering of the potential energy, while those characterized by $\nabla^{2} \rho_{\mathrm{BCP}}>0$ are dominated by a local excess in the kinetic energy.

Cremer and Kraka proposed to evaluate the total electronic energy density $[H(\mathbf{r})=G(\mathbf{r})+$ $\nu(\mathbf{r})]$ at the BCP, since in this manner the kinetic and potential energy densities have equal weights [42]:

$$
H_{\mathrm{BCP}}=G_{\mathrm{BCP}}+\nu_{\mathrm{BCP}}
$$

EQuation II

$H_{\mathrm{BCP}}$ is negative for interactions with significant sharing of electrons and its magnitude is a measure of 'covalency'.

\section{- QTAIM integrated multi-atomic property (delocalization index)}

A measure of the number of electron pairs shared between two atoms in a molecule is given by integrating the exchange density over each of the two atomic basins $A$ and $B$. For a closed-shell 
molecule, an electron delocalization index, $\delta(A, B)$, between atoms $A$ and $B$ is defined as [43]:

$$
\delta(A, B)=2\left|F^{\alpha}(A, B)\right|+2\left|F^{\beta}(A, B)\right|
$$

EQUATION 12

where

$$
\begin{aligned}
& F^{\sigma}(A, B) \\
& =-\sum_{i} \sum_{j} \int_{A} d \mathbf{r}_{1} \int_{B} d \mathbf{r}_{2}\left\{\phi_{i}^{*}\left(\mathbf{r}_{1}\right) \phi_{j}\left(\mathbf{r}_{1}\right) \phi_{j}^{*}\left(\mathbf{r}_{2}\right) \phi_{i}\left(\mathbf{r}_{2}\right)\right\} \\
& =-\sum_{i} \sum_{j} S_{i j}(A) S_{j i}(B)
\end{aligned}
$$

EQUATION 13

is the Fermi correlation, $S_{i j}(\Omega)=S_{j i}(\Omega)$ is the overlap integral of two orbitals over $\Omega$, and $\sigma$ is the spin $(\alpha$ or $\beta)$.

If both integrations in Equation 13 are performed over the same atom, $A$, the result is the total Fermi correlation for the electrons in $A$ with a limiting value of $-N^{\sigma}(A)$, the negative of the $\sigma$-spin population of atom $A$. This limit is reached in the hypothetical case whereby the electrons in $A$ are totally localized within $A$ and are not shared with any other atom in the molecule. This limit is approached, but never exactly reached, in closed-shell interactions, such as ionic bonding. It follows that a localization index $\Lambda(A)$ can be defined as:

$$
\Lambda(A, A)=\left|F^{\alpha}(A, A)\right|+\left|F^{\beta}(A, A)\right|
$$

EQUATION 14

In general $\left|F^{\alpha}(A, A)\right|<N^{\alpha}(A)$, which means that electrons in $A$ exchange to a degree or another with electrons in other atoms, for example, $B$, $C$ and $D$. In other words, electrons are always delocalized between atoms to a certain extent.

Localization and delocalization indices always sum to $N$ and provide a bookkeeping of the whereabouts of electrons in a molecule. Thus, the total electron population of an atom $\Omega, N(\Omega)$, is related to its localization index and the sum of its delocalization indices to all other atoms in the molecule (bonded or not) by:

$$
N(\Omega)=\Lambda(\Omega)+\frac{1}{2} \sum_{\Omega \neq \Omega^{\prime}} \delta\left(\Omega, \Omega^{\prime}\right)
$$

EQUATION 15

If $A$ and $B$ are bonded, that is, share a bond path, a BCP and an interatomic surface, then $\delta(A, B)$ measures the bond order when electron pairs are equally shared (i.e., when charge transfer is not significant) [43].

Because it is well known that $\rho_{\mathrm{BCP}}$ and bond order are exponentially related [26], it has been

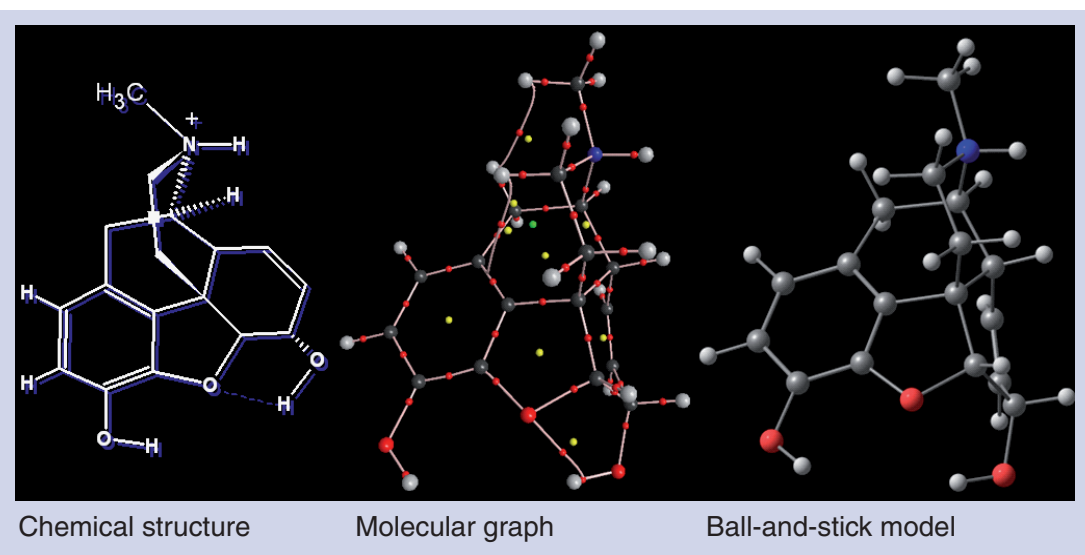

Figure 3. Chemical structure, molecular graph and a balls-and-stick representation of the optimized geometry of a protonated morphine molecule. The molecular graph is composed of the set of bond paths connecting bonded atoms (the small red dot on each bond path is the bond critical point).

The unconnected dots enclosed by rings are the ring critical points.

suggested to use the following equation to calibrate the delocalization index as a measure of bond order and the electron density at the BCP [44]:

$$
\delta(A, B)=\exp \left[a\left(\rho_{\mathrm{BCP}}-b\right)\right]
$$

EQUATION 16

This equation allows the delocalization index (not normally accessible from experiment) to be estimated on the basis of measured $\mathrm{x}$-ray electron-density distributions from the values of $\rho_{\mathrm{BCP}}$. Correlating the experimentally determined $\rho_{\mathrm{BCP}}$ values for the carbon-carbon bonds in the hormone molecule estrone with calculated delocalization indices yields [45]:

$$
\delta\left(C, C^{\prime}\right)=\exp \left\{4.7427 \times\left[\rho_{\mathrm{BCP}}(\text { in a.u. })-0.2538\right]\right\}
$$

EQuation 17

with an $r^{2}=0.939$.

\section{Analysis of the MEP}

The most fundamental levels at which molecular similarity can be described is the level of the electron density, $\rho(\mathbf{r})$, and that of the MEP, symbolized by $V(\mathbf{r})$. The MEP is derived from the total charge density $\left[\rho_{\text {total }}(\mathbf{r})=\sum_{\alpha} Z_{\alpha} \delta\left(R_{\alpha}-\mathbf{r}\right)-\rho(\mathbf{r})\right]$, that is, the discrete charge density due to the pointlike nuclei plus the diffuse electron density. The MEP is calculated from:

$$
V(\mathbf{r})=\sum_{A} \frac{Z_{A}}{\left|\mathbf{R}_{A}-\mathbf{r}\right|}-\int \frac{\rho\left(\mathbf{r}^{\prime}\right)}{\left|\mathbf{r}^{\prime}-\mathbf{r}\right|} d \mathbf{r}^{\prime}
$$

\section{EQuation 18}

where $Z_{A}$ is the charge of nucleus $A$ at a location given by the position vector $\mathbf{R}_{A}$, and $\rho(\mathbf{r})$ is the 
electron density. Equation 18 is the solution of the Poisson equation for the total charge density. However, since the electron density $\rho(\mathbf{r})$ determines the external potential $v(\mathbf{r})$ (the first term on the right-hand side of Equation 18) through mapping (1), the electron density and the MEP are uniquely mapped to one another as well. It is worth emphasizing here that both the electron density and the electrostatic potential are experimentally accessible physical fields through the refinement of high-resolution $\mathrm{x}$-ray diffraction data $[30,31]$.

\section{Use of QTAIM bond properties in the constructions of QSAR/QSPR}

Molecular similarity can be inferred from the substituent effects on a common scaffold, on the basis of the conjecture that similar groups exert similar substituent effects. An example of a quantitative experimental measure of substituent effects is the Hammett substituent constant [46]. The Hammett constant is defined from its namesake equation correlating the equilibrium constants $K$ of substituted benzoic acids to the unsubstituted molecule:

$$
\sigma=\log \frac{K_{S}}{K_{H}}=p K_{a, H}-p K_{a, S}
$$

EQUATION 19

where $K_{H}$ and $K_{S}$ are the ionization constants of unsubstituted and substituted benzoic acid, respectively. The Hammett constant is, thus, an experimental descriptor obtained from the measurement of $p K_{a}$ values. This descriptor is very well predicted from theoretical calculation within the framework of the approach termed, quantum topological molecular similarity (QTMS), developed by Popelier and co-workers [47-54].

In a nutshell, QTMS summarizes the description of a molecule in terms of its bond properties. In this approach, the electron density is only sampled at the BCPs (instead of being wiped through all space) where it is described in detail. This sampling affords considerable advantages including speed, simplicity, focus on chemically relevant region (chemical bonds) and bypassing the dominance of the chemically irrelevant nuclear regions. Furthermore, QTMS is not plagued with the molecular alignment problem, a conceptual and practical advantage over formulae of the form of Equation 2. A limitation of QTMS is its lack of ability to distinguish between optical isomers, unlike Equation 2.

In QTMS, an abstract multidimensional mathematical space is constructed from the bond properties determined at the $\mathrm{BCP}$, one dimension per property. The similarity of two molecules (that have at least one common moiety) is then given by the Eucledian distance between them in this abstract space. Examples of bond descriptors include $\rho_{\mathrm{BCP}}, \nabla^{2} \rho_{\mathrm{BCP}}, \varepsilon, \lambda_{1}, \lambda_{2}, \lambda_{3}, G_{\mathrm{BCP}}, H_{\mathrm{BCP}}$, $R_{e}$ (the equilibrium bond length). For further illustration, if the BCP property set includes only three descriptors, say $\rho_{\mathrm{BCP}}, \nabla^{2} \rho_{\mathrm{BCP}}$ and $\varepsilon$, then the mathematical bond property space is three-dimensional and the Euclidean distances between two molecules $A$ and $B$ is the sum of all the distances of corresponding bonds, that is,

$$
\begin{aligned}
& d(A, B)= \\
& \sum_{i \in A} \sum_{j \in B} \sqrt{\left(\rho_{B C P}^{A}-\rho_{B C P}^{B}\right)^{2}+\left(\nabla^{2} \rho_{B C P}^{A}-\nabla^{2} \rho_{B C P}^{B}\right)^{2}+\left(\varepsilon^{A}-\varepsilon^{B}\right)^{2}} \\
& \text { EQuation } 20
\end{aligned}
$$

where $\rho_{\mathrm{BCP}}, \nabla^{2} \rho_{\mathrm{BCP}}$ and $\varepsilon$ are each transformed into a dimensionless relative scale to ensure dimensional homogeneity.

In the first paper on QTMS, Popelier showed that the Euclidean distance defined in Equation 20 is, in principle, capable of detecting the 'active center' automatically. Thus, Popelier has shown that the experimental $\sigma$-sequence with respect to $p$-aminobenzoic acid $\left(\mathrm{NH}_{2}>\mathrm{OCH}_{3}\right.$ $>\mathrm{CH}_{3}>\mathrm{H}>\mathrm{F}>\mathrm{C}>\mathrm{CN}>\mathrm{NO}_{2}$ ) is reproduced if the bonds in the 'active center' (and only the bonds in the 'active center') are included in the definition of the Euclidean distance, namely, the $\mathrm{O}(-) \mathrm{H}, \mathrm{C}(=) \mathrm{O}$, and $\mathrm{C}(-) \mathrm{COOH}$ bonds (the parentheses indicate the bond included in the definition). The inclusion of any other bond(s) from the ring or from the substituent, $S$, in the definition of the Euclidean distance causes the predicted sequence to depart from the experimental sequence to a degree or another, as can be seen from TABLE I. The worst agreement of the predicted and experimental sequence is that when all 15 bonds are included in the calculation of the distance. The ability of QTMS to 'point' at the 'active center' is an exciting and promising result as it suggests that information about the 'active center' is encoded in the electron densityderived bond properties defining it. Bond properties treated by QTMS appear, thus, to provide the basis for robust QSAR/QSPR modeling of series of related compounds and, also, engender the possibility of an automatic detection of active centers.

A corollary of the results described in the previous paragraph is that one could rely on the properties of the active center, when known, in cases 
Table 1. Prediction of the $\sigma$-constants ranking of $p$-substituted benzoic acid relative to $p$-amino benzoic acid from the gradual addition of bonds into the summation defining the Euclidean distance (EQUATION 20).

\begin{tabular}{|c|c|c|c|c|c|c|c|c|c|}
\hline Experimental sequence & & $\mathrm{NH}_{2}$ & $\mathrm{OCH}_{3}$ & $\mathrm{CH}_{3}$ & $\mathbf{H}$ & $\mathbf{F}$ & $\mathrm{Cl}$ & $\mathrm{CN}$ & $\mathrm{NO}_{2}$ \\
\hline $\begin{array}{l}\text { Quantum topological molecular } \\
\text { similarity sequences }\end{array}$ & $\begin{array}{l}\text { Number of bond } \\
\text { critical points }\end{array}$ & & & & & & & & \\
\hline $\mathrm{OH}$ & 1 & $\mathrm{NH}_{2}$ & $\mathrm{OCH}_{3}$ & $\mathrm{CH}_{3}$ & H & $\mathbf{F}$ & $\mathrm{Cl}$ & $\mathrm{CN}$ & $\mathrm{NO}_{2}$ \\
\hline$C=0$ & 1 & $\mathrm{NH}_{2}$ & $\mathrm{OCH}_{3}$ & $\mathrm{CH}_{3}$ & H & $\mathbf{F}$ & $\mathrm{Cl}$ & $\mathrm{CN}$ & $\mathrm{NO}_{2}$ \\
\hline $\mathrm{COOH}$ & 3 & $\mathrm{NH}_{2}$ & $\mathrm{OCH}_{3}$ & $\mathrm{CH}_{3}$ & H & $\mathbf{F}$ & $\mathrm{Cl}$ & $\mathrm{CN}$ & $\mathrm{NO}_{2}$ \\
\hline $\mathrm{C}-\mathrm{COOH}$ & 4 & $\mathrm{NH}_{2}$ & $\mathrm{OCH}_{3}$ & $\mathrm{~F}$ & $\mathrm{CH}_{3}$ & $\mathrm{Cl}$ & H & $\mathrm{CN}$ & $\mathrm{NO}_{2}$ \\
\hline $\mathrm{C}_{6}$ & 6 & $\mathrm{NH}_{2}$ & $\mathrm{CN}$ & $\mathrm{CH}_{3}$ & $\mathrm{OCH}_{3}$ & $\mathrm{H}$ & $\mathrm{Cl}$ & $\mathrm{NO}_{2}$ & $\mathrm{~F}$ \\
\hline $\mathrm{C}_{6} \mathrm{H}_{4}$ & 10 & $\mathrm{NH}_{2}$ & $\mathrm{CH}_{3}$ & $\mathrm{OCH}_{3}$ & $C N$ & $\mathrm{H}$ & $\mathrm{Cl}$ & $\mathrm{NO}_{2}$ & $\mathrm{~F}$ \\
\hline $\mathrm{C}_{6}-\mathrm{COOH}$ & 10 & $\mathrm{NH}_{2}$ & $\mathrm{OCH}_{3}$ & $\mathrm{CH}_{3}$ & $\mathrm{Cl}$ & $\mathrm{CN}$ & $\mathrm{H}$ & $\mathrm{NO}_{2}$ & $\mathrm{~F}$ \\
\hline $\mathrm{C}_{6} \mathrm{H}_{4}-\mathrm{COOH}$ & 14 & $\mathrm{NH}_{2}$ & $\mathrm{CH}_{3}$ & $\mathrm{CH}_{3}$ & $\mathrm{Cl}$ & $\mathrm{H}$ & $C N$ & $\mathrm{NO}_{2}$ & $\mathrm{~F}$ \\
\hline $\mathrm{S}-\mathrm{C}_{6} \mathrm{H}_{4}-\mathrm{COOH}^{(\ddagger)}$ & 15 & $\mathrm{NH}_{2}$ & $C N$ & $\mathrm{H}$ & $\mathrm{OCH}_{3}$ & $\mathrm{CH}_{3}$ & $\mathrm{NO}_{2}$ & $\mathrm{~F}$ & $\mathrm{Cl}$ \\
\hline
\end{tabular}

where the molecules are very different and do not share any common skeleton but that center. Two striking examples are shown in Figure 4. In one plot (FIGURE 4A), the $p K_{a}$ has been predicted on the basis of only the bonds constituting the carboxylic group of a series of 40 small, unrelated
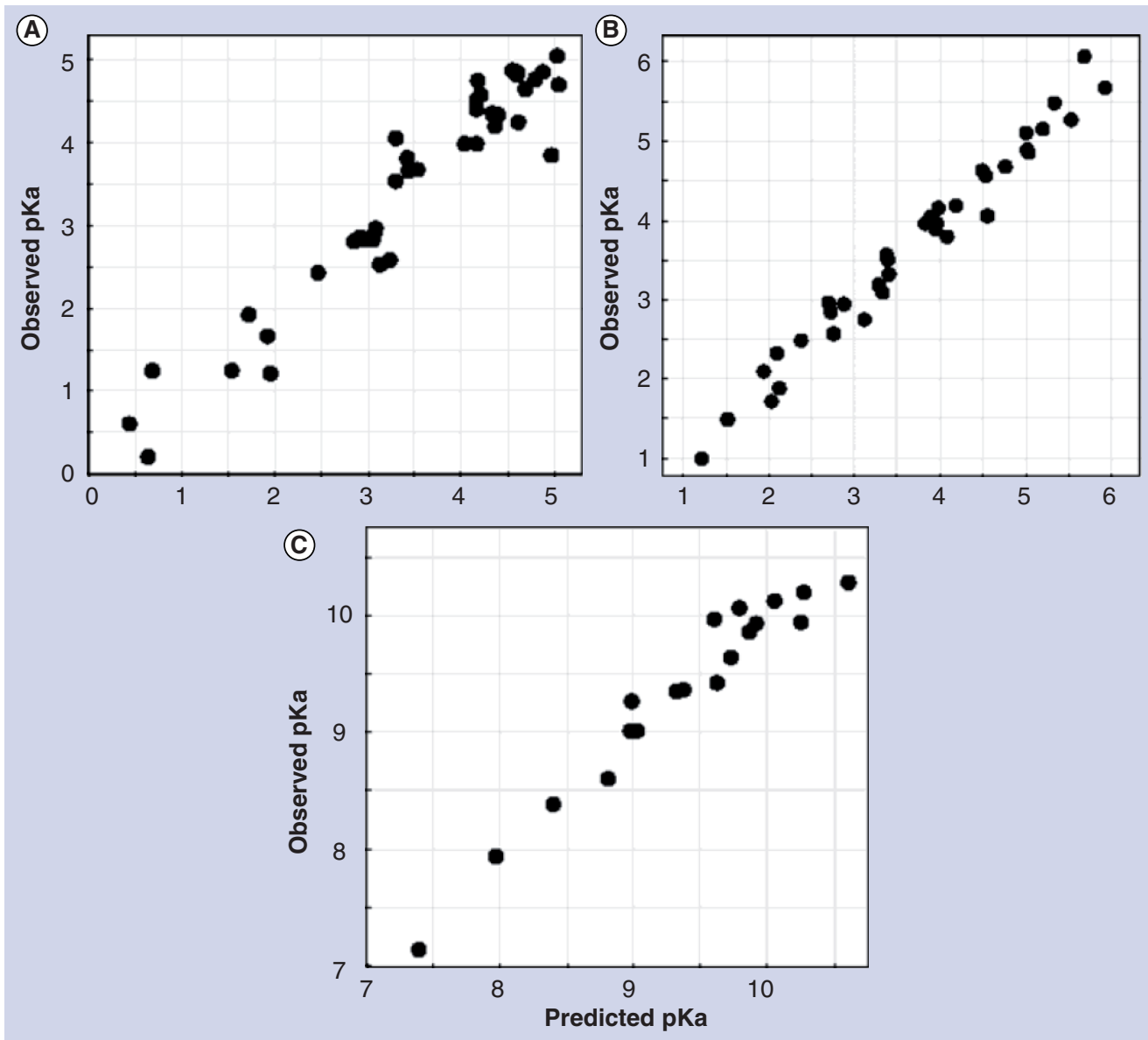

Figure 4. (A) Correlation of observed and predicted $p K_{a} s$ of a set of 40 generally unrelated small organic carboxylic acids. (B) Correlation of observed and predicted $p K_{a} s$ of 36 substituted anilines. (C) Correlation of observed and predicted $p K_{a} s$ of 19 substituted phenols.

Reproduced with permission from [51]. @ PLA Popelier and the American Chemical Society. 
organic acids [51]. In this study, partial least-square regression has been used to choose the most important bond critical point descriptors, four per bond, to a total of 12 descriptors per acid molecule. In contrast to these unrelated molecules, the two remaining plots (FIgures 4B \& C) illustrate the usefulness of QTMS to predict the $p K_{a}$ values of a series of closely related substituted anilines (Figure 4B) and phenols (Figure 4C). In the two latter cases, all bonds in the common fragment are included in the correlation. Collectively, the three plots in Figure 4 cover a $p K_{a}$ range from the vicinity of 0.4 to as high as 10.6. Chaudry and Popelier [51] showed the robustness of QTMS predictive statistics by going beyond $r^{2}$ to the 'leave one out' cross-validated correlation coefficient $q^{2}$ to eliminate chance correlations. The $\left(r^{2} ; q^{2}\right)$ values for a series of 40 carboxylic acids, 36 anilines, and 19 phenols were found to be $(0.920 ; 0.891)$, $(0.974 ; 0.953)$, and $(0.952 ; 0.884)$, respectively. These results outperform traditional descriptors such as Hammett substituent constants [51]. More recently, Haw et al. extended the use of QTMS along with partial least-square regression and machine learning techniques to predict, with very high accuracy, the proton affinities of 125 pyridine derivatives [53].

\section{Use of QTAIM atomic properties in the constructions of QSAR/QSPR}

- Prediction of $p K_{a}$ from atomic energies of the acidic hydrogen

In the acid dissociation:

$$
\mathrm{HA}+\mathrm{H}_{2} \mathrm{O} \rightleftharpoons \mathrm{H}_{3} \mathrm{O}^{+}+\mathrm{A}^{-}
$$

the $p K_{a}$ is given by

$$
p K_{a}=\frac{\Delta G^{0}}{2.303 R T}
$$

Equation 21

or equivalently,

$$
\ln K_{a}=\frac{-\Delta G^{0}}{R T}
$$

EQUATION 22

Equation 23

From a series of reasonable statistical mechanical approximations, Adam has derived an approximate relationship between $p K_{a}$ of a weak acid and the QTAIM atomic energy of the acidic (dissociating) proton [55]. To give the reader an appreciation of the physical bases of Adam's approach, we briefly retrace here the essential steps, approximations and results he reported [55].
Let $U_{J}^{0}$ be the molar dissociation energy, that is, the molar energy difference between the lowest vibrational state of a molecule $A_{J}$ and the sum of the energies of the ground-state separate atoms composing it. EQuation 23, when applied to equilibrium (EQuation 21), can be re-expressed in terms of $U_{J}^{0}$ and the standard molar partition functions $q_{J}^{0}$ of the products and reactants as:

$$
\ln K_{a}=\frac{\left(U_{H_{A}}^{0}+U_{\mathrm{H}_{2} \mathrm{O}^{-}}^{0} U_{A^{-}}^{0}-U_{\mathrm{H}_{3} \mathrm{O}^{+}}^{0}\right)}{R T}+\ln \left(\frac{q_{\mathrm{H}_{3} \mathrm{O}^{+}}^{0}}{q_{\mathrm{HA}}^{0}} \frac{q_{A^{-}}^{0}}{q_{H_{2} \mathrm{O}}^{0}}\right)
$$

Upon rearrangement, this equation can be re-written as:

$$
p K_{a}=\frac{U_{A^{-}}^{0}-U_{H A}^{0}}{2.303 R T}-\underbrace{\log \left(\frac{q_{A-}^{0}}{q_{H A}^{0}}\right)}_{\approx 0} \underbrace{\frac{U_{H_{3} \mathrm{O}^{+}}^{0}-U_{H_{2} \mathrm{O}}^{0}}{2.303 R T}-\log \left(\frac{q_{H_{3} \mathrm{O}^{+}}^{0}}{q_{H_{2} \mathrm{O}}^{0}}\right)}_{\text {EQUATION } 25}
$$

Adam assumed that the similarity of the partition functions of the acid in the dissociated or unionized form ( $\mathrm{A}^{-}$and HA, respectively) is such that $q_{A^{-}}^{0} \approx q_{H A}^{0}$. As a result of this assumption, the second term in Equation 25 vanishes $(\log 1=0)$. The accuracy of this approximation is expected to increase with the size of the acid molecule, as noted by Adam [55]. Furthermore, while the third and fourth terms in EQuation $\mathbf{2 5}$ are temperaturedependent, they are independent of the nature of the acid since these include the dissociation energies and partition functions of the hydronium ion and water but not of the acid. Adam calls this constant $C^{\prime}$. Equation $\mathbf{2 5}$ then simplifies to:

$$
p K_{a}=\frac{U_{A^{-}}^{0}-U_{H A}^{0}}{2.303 R T}+C^{\prime}
$$

EQUATION 26

Realizing that $U_{J}^{0}$ is related to the energy of dissociation (from the bottom of the well, rather than from the zero-point vibrational energy level) $E_{J}$ by

$$
E_{J}=U_{J}^{0}-E_{J}^{Z P E}+\sum_{i=1}^{n_{J}} E_{i}
$$

EQuATION 27

where the last term is the sum of the electronic energies of the separated atoms in their ground states, it follows that:

$$
U_{A^{-}}^{0}-U_{H A}^{0}=E_{A^{-}}-E_{H A}+\underbrace{E_{\text {constant }}^{E_{H}}+\underbrace{E_{A^{-}}^{Z P E}-E_{H A}^{Z P E}}_{\begin{array}{l}
\text { very small and } \\
\text { approximately } \\
\text { constant }
\end{array}}}_{\approx \text { constant }}
$$

EQUATION 28 
where the difference $E_{A^{-}}^{Z P E}-E_{H A}^{Z P E}$ is expected to be very small and approximately constant for a homologous series of acids, and $E_{\mathrm{H}}$, the energy of a free ground-state hydrogen atom is of course constant. Thus, it can be reasonably assumed that the last three terms in Equation 28 are approximately constant. With this result, Equation 26 is recast in terms of electronic energies:

$$
p K_{a}=\frac{E_{A-}-E_{H A}}{2.303 R T}+C
$$

Equation 29

where the new constant terms have been combined with $C^{\prime}$ to yield $C$.

At this point, Adam uses QTAIM's definition of atomic energies to simplify Equation 29. He identifies the difference between the electronic energies of the anion and neutral acid molecules as approximately equal to the atomic energy of the acidic (dissociating) hydrogen atom, that is:

$$
E_{A-}-E_{H A} \approx-E(\mathrm{H})
$$

EQUATION 30

where $E(\mathrm{H})$ is the atomic energy of the acidic hydrogen in the undissociated acid molecule HA. This last approximation is also expected to be more accurate for a larger molecular size.

With this last approximation, the remarkable final equation derived by Adam is:

$$
p K_{a}=\frac{-E(\mathrm{H})}{2.303 R T}+C
$$

Equation 31

This final result can be applied to either gas- or solution-phase $p K_{a}$ depending on the underlying electronic-structure determination of the wavefunction of the acid. Solution-phase $p K_{a}$ can be calculated from the atomic energy of the acidic hydrogen obtained from a wavefunction calculated in solution whether discrete or continuum such as the polarized continuum model.

Adam tested his suggested relationship by a least-squares linear regression analysis of computed versus experimental values. As an example, the reported tabulation with the largest number of entries, 53 acids of various kinds, follows the following fitted equation:

$$
\begin{aligned}
& p K_{a}(\text { exptl })= \\
& -0.1725\left(\text { mol kJ }^{-1}\right) \times E(\mathrm{H})\left(k J \text { mol }^{-1}\right) \underbrace{-150.486}_{C}
\end{aligned}
$$

with an $r^{2}=0.991$.
We have plotted the data reported in [55] in Figure 5. The excellent agreement between the calculated and experimental $p K_{a}$ values is shown in Figure 5A, and Figure 5B shows the strong statistical correlation between the QTAIM atomic energies of the acidic hydrogen and the $p K_{a}$. TABLE 2 summarizes the remainder of the statistical correlations reported in this important paper [55]. One can infer from the strong predictive power of EQuation $3 \mathbf{I}$ that the statistical mechanical assumptions made to derive it are sound and physically meaningful.

\section{- Modeling of partial molar volumes}

In a two-component system, the partial molar volume is defined as the rate of change of the volume of the solution with respect to the number of moles of solute at a given temperature $(T)$, pressure $(P)$, and number of moles of solvent $\left(n_{\text {solvent }}\right)$ at infinite dilution, that is:

$$
V^{0}=\left[\frac{\partial V}{\partial n_{\text {solute }}}\right]_{T, P, n_{\text {solvent }}}
$$

EQuation 33

$V^{0}$ is not the quantity directly measurable, one rather measures the apparent partial molar volume $\left(V_{a p p}^{0}\right)$. The equality of the two quantities, $V^{0}=V_{a p p}^{0}$, is achieved only at infinite dilution, and, hence, experimental partial molar volumes are obtained through extrapolation.

At infinite dilution, $V^{0}$ results from two principal contributions: a positive intrinsic volume contribution $\left(V_{\text {intrinsic }}^{0}\right)$ due to the volume occupied by the solute and which pushes the solvent molecule from the cavity it occupies, and a generally negative electrostriction contribution $\left(V_{\text {electrostriction }}^{0}\right.$ especially when the solvent is polar. The latter effect is due to the attraction of the solute to the oppositely charged ends of the surrounding solvent molecules, and hence has a net effect of collapsing the volume of the solution. The partial molar volume of an organic molecule can thus be written as [56]:

$$
V^{0}=V_{\text {intrinsic }}^{0}+V_{\text {electrostriction }}^{0}
$$

Equation 34

Since QTAIM treats atoms in a molecule on the same footing as the parent molecule itself, correspondingly atomic (or group) contributions to the partial molar volume of a molecule can be anticipated. The partial molar volume contribution of an atom in a molecule is itself made up of atomic intrinsic and electrostriction terms: 
Table 2. Summary of the results reported in [55] obtained using Equation 3 I in the prediction of the $p K_{a}$ vlaues of several series of compounds.

\begin{tabular}{|c|c|c|c|c|}
\hline Compound families & $n^{\dagger}$ & $r^{2 \ddagger}$ & $\begin{array}{l}\text { Average } \\
\text { deviation }\end{array}$ & $\begin{array}{l}\text { Maximum } \\
\text { absolute } \\
\text { deviation }\end{array}$ \\
\hline Aliphatic carboxylic acids & 19 & 0.98 & 0.10 & 0.34 \\
\hline Substituted phenols & 20 & 0.92 & 0.21 & 0.6 \\
\hline Substituted anilinium ions & 12 & 0.96 & 0.14 & 0.27 \\
\hline 3,4-substituted pyridinium ions & 11 & 0.97 & 0.33 & 0.68 \\
\hline $\begin{array}{l}\text { Hydrated aliphaticacids, substituted } \\
\text { benzoic acids and substituted phenols }\end{array}$ & 53 & 0.99 & 0.22 & 0.62 \\
\hline
\end{tabular}

$$
V^{0}(\Omega)=V_{\text {intrinsic }}^{0}(\Omega)+V_{\text {electrostriction }}^{0}(\Omega)
$$

EQuATION 35

In Equation 35, the atomic intrinsic volume is modeled by the van der Waals' volume, that is, the volume enclosed by the union of one or more zero-flux surface(s) with an outer isodensity envelope. The outer envelope that is often chosen is the one with $\rho=0.001$ au as this envelope usually encloses more than $99 \%$ of the electron population of a molecule and correlates very well with the experimental van der Waals' radii [26].

The atomic electrostriction volume in EQuation 35 is postulated to be a function of the magnitude of the atomic charge, since both positive and negative charges can attract solvent water molecules. This contribution is thus modeled by the sum of the unsigned atomic charges on all the atoms in the molecule, a sum termed the charge separation index (CSI), defined as [57]:

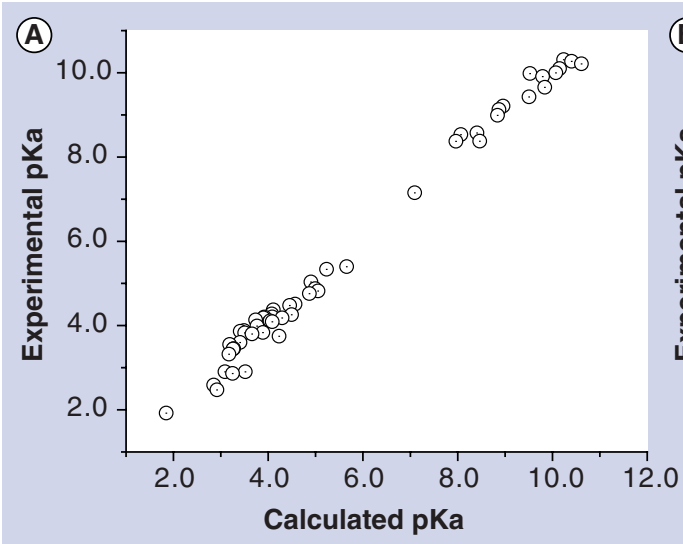

$$
\mathrm{CSI}=\sum_{\Omega}|q(\Omega)|
$$

EQuATION 36 where $q(\Omega)$ is the charge of atom $\Omega$. It is clear from this definition that the CSI provides an overall measure of local polarity of the molecule.

Modeling the partial molar volumes of the 20 naturally occurring amino acids

Figure 6 displays the atomic charges on the neutral side chains of a nonpolar amino acid (leucine, [Leu]) juxtaposed next to a polar amino amino acid (asparagine [Asn]). As expected for neutral side chains, the total charge on each side chain (the sum of the atomic charges) is close to zero $(+0.07$ and +0.04 e, respectively), implying minimal charge transfer to the common $-\mathrm{C}_{\alpha} \mathrm{H}_{\alpha}\left(\mathrm{NH}_{2}\right) \mathrm{COOH}$ group. However, the CSI, as defined in Equation 36, strongly discriminates a polar from a nonpolar side chain (0.91 and 5.60 e for Leu and Asn, respectively).

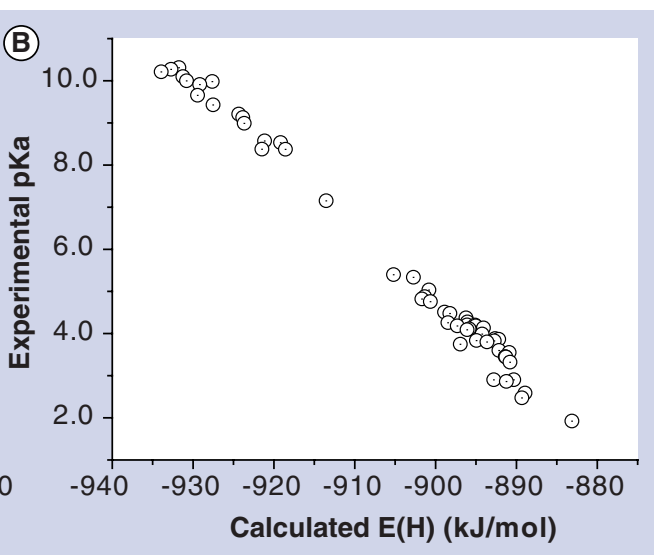

Figure 5. (A) Correlation of experimental and $p K_{a}$ values predicted from EQuATION 32 of a heterogeneous set of 53 small carboxylic acids. (B) Correlation of experimental $p K_{a}$ values of the same set of 53 acids with the quantum theory of atoms in molecules atomic energy of the acidic (dissociating) hydrogen atom in the undissociated acid (HA).

Data from [55]. 


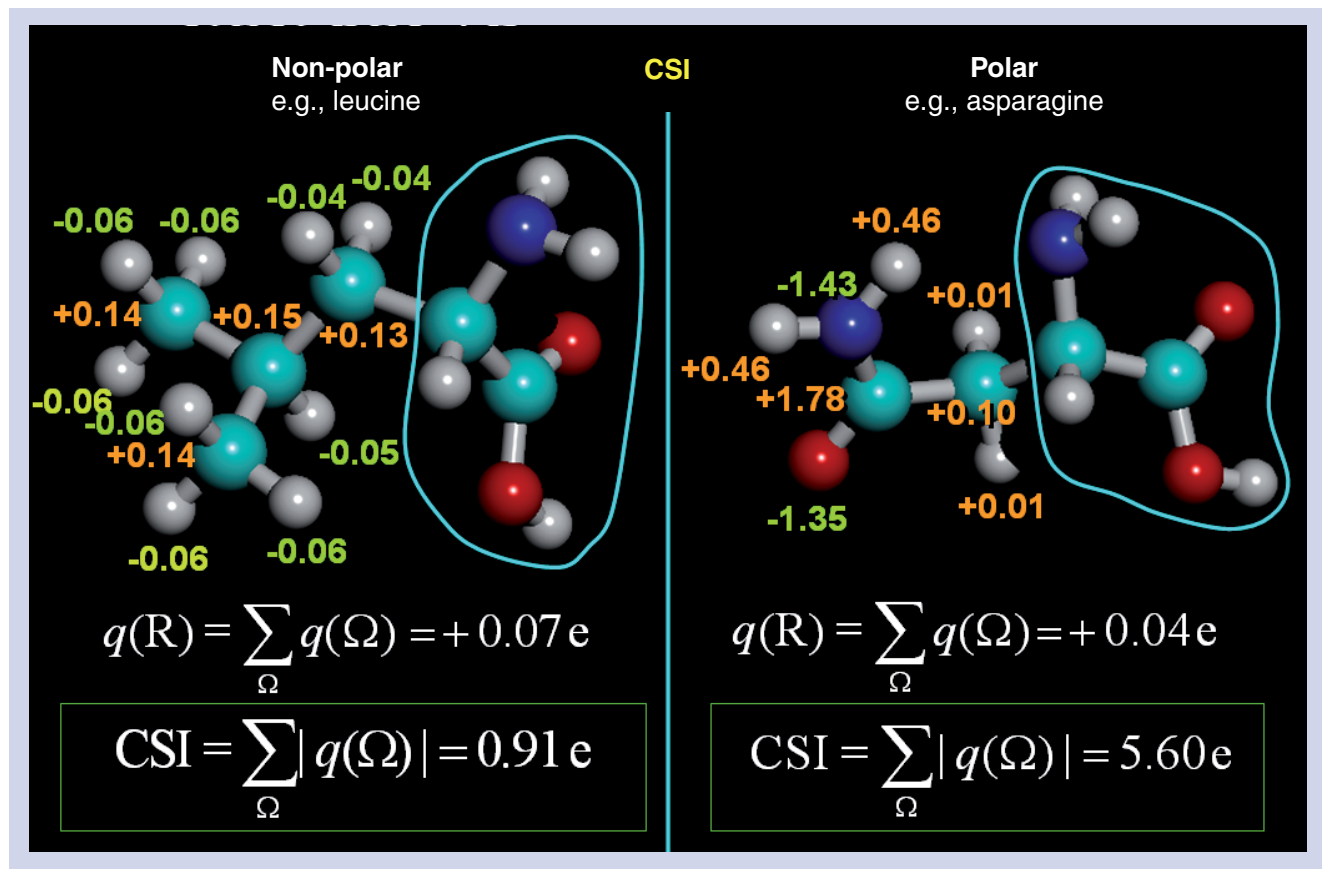

Figure 6. Charge separation index as a measure of polarity of the side chains of two prototypical amino acids: one nonpolar (leucine, Leu) and another polar (asparagine, Asn). The figure displays the optimized geometries of the two molecules labeled with quantum theory of atoms in molecules atomic charges on the side chains of the two amino acids. The two side chains are nearly neutral as the sums of the charges indicate. However, the sums of the magnitudes (unsigned values) of the atomic charges, the CSI, clearly discriminate between a polar and a nonpolar side chain. The circled portion, $-\mathrm{C}_{\alpha} \mathrm{H}_{\alpha}\left(\mathrm{NH}_{2}\right) \mathrm{COOH}$, is not considered, being common to all amino acids.

CSI: Charge separation index.

Experimental partial molar volumes for the series of the 20 genetically encoded amino acids side chains, each capped with a hydrogen atom, were obtained from the literature [58]. Since all natural amino acids contain the $-\mathrm{C}_{\alpha} \mathrm{H}_{\alpha}\left(\mathrm{NH}_{2}\right)$ $\mathrm{COOH}$ group, it is assumed that the additive contribution of this group to the partial molar volume is approximately constant at a given temperature and can be ignored [59], placing the focus exclusively on the side chains $(R)$. The modeling of the partial molar volume results in the regression equation [59]:

$V_{\mathrm{AA}}^{0}=37.250+0.098 \times V(\mathrm{vdW})_{R}-0.884 \times \mathrm{CSI}_{R}$ $\left[r^{2}=0.978, s=3.887, n=20\right]$

EQuation 37 where $V(\mathrm{vdW})_{R}$ is the van der Waals' volume and $\mathrm{CSI}_{R}$ is the CSI of the side chain $R$. The second term of Equation 37 represents the solvent cavity or excluded volume formed due to the presence of the side chain and, hence, contributes positively to the partial molar volume. The third term is negative since it represents the shrinkage in the solvent architecture due to electrostriction. It is worth emphasizing that the above correlation is characterized by $r^{2}=0.978$ yet only two parameters are used to fit 20 data points. The correlation between the predicted and experimental partial molar volumes is graphically displayed in Figure 7A.

A similar correlation is also found for the individual group contributions to the partial molar volumes of the amino acids [59]:

$$
\begin{aligned}
& V_{G}^{0}=-0.925+0.127 \times V(\mathrm{vdW})_{G}-2.456 \times \mathrm{CSI}_{G} \\
& {\left[r^{2}=0.983, s=1.122, n=8\right]}
\end{aligned}
$$

EQUATION 38

where $V_{G}^{0}$ is the experimental group contributions to the partial molar volume (from [58]), and $V(\mathrm{vdW})_{G}$ and $\mathrm{CSI}_{G}$ are group van der Waals' volume and CSI, respectively. This correlation is displayed graphically in Figure 7B.

Modeling the partial molar volumes of the five nucleic acid bases

The electron densities of the five free nucleic acid bases adenine, guanine, cytosine, thymine and uracil, were also analyzed using QTAIM (for technical details of electronic structure 
calculations, see [60]). Aqueous partial molar volumes of four of the free bases (except guanine) and all of the five nucleosides were accurately measured by Lee and Chalikian [61] at two different temperatures $\left(18\right.$ and $\left.55^{\circ} \mathrm{C}\right)$. To obtain an estimate of the group contribution of the sugar moiety to the partial molar volume, the reported partial molar volumes of the bases are subtracted from the corresponding partial molar volumes of the nucleosides [60]:

$$
V_{\text {sugar }}^{0} \approx V_{\text {nucleoside }}^{0}-V_{\text {base }}^{0}
$$

Equation 39

The calculated group contribution of the sugar moiety to the partial molar volume of the nucleosides according to Equation 39 shows a remarkable transferability, being independent of the base to which it is attached within experimental uncertainties (TABLE 3). The sugar contributes $\left\langle V_{\text {sugar }}^{0}\right\rangle 80.4 \pm 1.0 \mathrm{~cm}^{3} \mathrm{~mol}^{-1}$ within the temperature range $\left(18-55^{\circ} \mathrm{C}\right)$. Using this transferable group contribution of the sugar moiety, the partial molar volume of guanine (not reported in [61]) is obtained from [60]:

$$
V_{\text {guanine }}^{0} \approx V_{\text {guanosine }}^{0}-\left\langle V_{\text {sugar }}^{0}\right\rangle
$$

Equation 40

TABLE 3 lists the experimental and predicted values of the partial molar volumes of the five nucleic acid bases in aqueous solutions. The calculation of the partial molar volumes of the bases was achieved using the van der Waals' volume $V(\mathrm{vdW})$ and the CSI as predictors, as was done for the amino acids. The least-square fitting yields the following regression equations at the two experimental temperatures [60]:

$V_{\text {base }}^{\mathrm{o}}\left(18^{\circ} \mathrm{C}\right)=0.09508 \times V(\mathrm{vdW})_{\text {base }}-1.25304 \times \mathrm{CSI}_{\text {base }}$ $\left[r^{2}=0.980, s=2.527, n=5(A, T, U, G, C)\right]$

EQUATION 4I

and

$V_{\text {base }}^{\mathrm{o}}\left(55^{\circ} \mathrm{C}\right)=0.1 .0050 \times V(\mathrm{vdW})_{\text {base }}-1.30930 \times \mathrm{CSI}_{\text {base }}$ $\left[r^{2}=0.967, s=4.895, n=5(A, T, U, G, C)\right]$

EQUATION 42 correlations that are displayed in FigurE 8, where $A$ is adenine, $T$ is thymine, $U$ is uracil, $G$ is guanine and $\mathrm{C}$ is cytosine.

Molecular vibrations increase the apparent volume of a molecule in solution and, hence, contributes a positive 'thermal volume' to the partial molar volume [61]. This thermal increase in the partial molar volumes is consistently recovered in the predicted partial molar volumes of the five bases as the temperature is raised from 18 to $55^{\circ} \mathrm{C}$ (Figure 8). It is concluded that a simple modeling based only on $V(\mathrm{vdW})$ and CSI appears to be sufficiently sensitive to capture much of the physics determining partial molar volumes, including its thermally induced vibrational increase.

- Modeling of the partitioning coefficient between two immiscible liquid phases

The partition coefficient is a dimensionless ratio of the equilibrium concentrations of a solute in two immiscible liquids brought into contact:

$$
P=\frac{C_{S_{1}}}{C_{S_{2}}}
$$

EQUATION 43

where $C_{S_{1}}$ and $C_{S_{2}}$ are the concentration of the given solute in solvents $s_{1}$ and $s_{2}$, usually octanol and water, respectively.

Partition coefficients are of paramount importance in medicinal chemistry as they determine the partitioning of a solute between an aqueous phase, such as the serum of blood, and biomembranes such as the blood-brain barrier. The central role of $P$ in medicinal chemistry can be illustrated by its correlation to the equilibrium constant of binding of organic molecules to proteins [62]:

$$
\log K=k_{2} \log P+k_{4}
$$

EQUATION 44

where $k_{2}$ and $k_{4}$ are constants, and $K$ is the equilibrium constant of the binding of the solute to the protein. The binding of drug molecules to bovine serum albumin is found to closely fit Equation 44 with $k_{2}=0.751 \pm 0.007$ and $k_{4}=2.301 \pm 0.15$, this fit constructed from 42 data points yields a standard error of 0.159 and an overall $r^{2}$ as high as 0.960 [63].

Often $K$ expresses the biological activity directly $K=1 / C$ where $C$ is the concentration of drug necessary to elicit a biological end point, for example $\mathrm{EC}_{50}, \mathrm{LD}_{50}$ and $\mathrm{IC}_{50}$. Sometimes $C$ is defined as the concentration of drug that yields a 1:1 complex with a given protein. Among important applications of Equation 44 is in the determination of the dose of drugs as the proportion of drug that is bound to serum albumin is unavailable for binding to its target receptor(s). 
Hansch is credited to have realized that Equation 44 represents an approximately linear segment of a more general parabolic relationship that emerges when a broader range of $\log P$ values is examined [64]. The interpretation of the parabolic correlation between biological activity and $\log P$ is as follows: a drug is must have a high enough affinity to the hydrophobic biological membrane to be able to dissolve and diffuse through it but this affinity must be low enough for the drug to eventually leave the membrane to the aqueous environment where it is intended to exert its therapeutic action.

Hansch's more general parabolic relationship is [64]:

$$
\log \frac{1}{C}=-k_{1}(\log P)^{2}+k_{2} \log P+k_{3} \sigma+k_{4}
$$

EQUATION 45

where, in addition to the first quadratic term, a third term proportional to Hammett substituent constant is added as well. The third term does not contribute for neutral molecules or when the change in the ionization state has little effect on the rate of diffusion through biomembranes, and is irrelevant for molecular libraries consisting of totally unrelated molecules [65]. When this third term is omitted, the equation takes its simpler form:

$$
\log \frac{1}{C}=-k_{1}(\log P)^{2}+k_{2} \log P+k_{4}
$$

EQUATION 46

The parabolic relationship described by Equation 45 or Equation 46 implies that there will be an optimal value for $\log P$, this value is often referred to as $\log P^{0}$ in the literature [66]. The activity of general anesthetics of the ethers family follows closely Equation 46 with $k_{1}=0.22$, $k_{2}=1.04$, and $k_{4}=2.16$ [66]. It is remarkable that, regardless of the chemical nature of an anesthetic, the value of $\log P^{0} \approx 2.3$ is consistently found to be a requirement for optimal biological activity [66].

While the motivation to model the partition coefficient of the 20 genetically encoded amino acid was different in the original literature (to simulate the effect of mutation on proteins folding) $[59,60]$, the accurate modeling of partition coefficients is of prime relevance to medicinal chemistry for the reasons outlined previously. This is what will be described in the remainder of this section, an analysis that has never been published before despite being based on previous calculations [59].

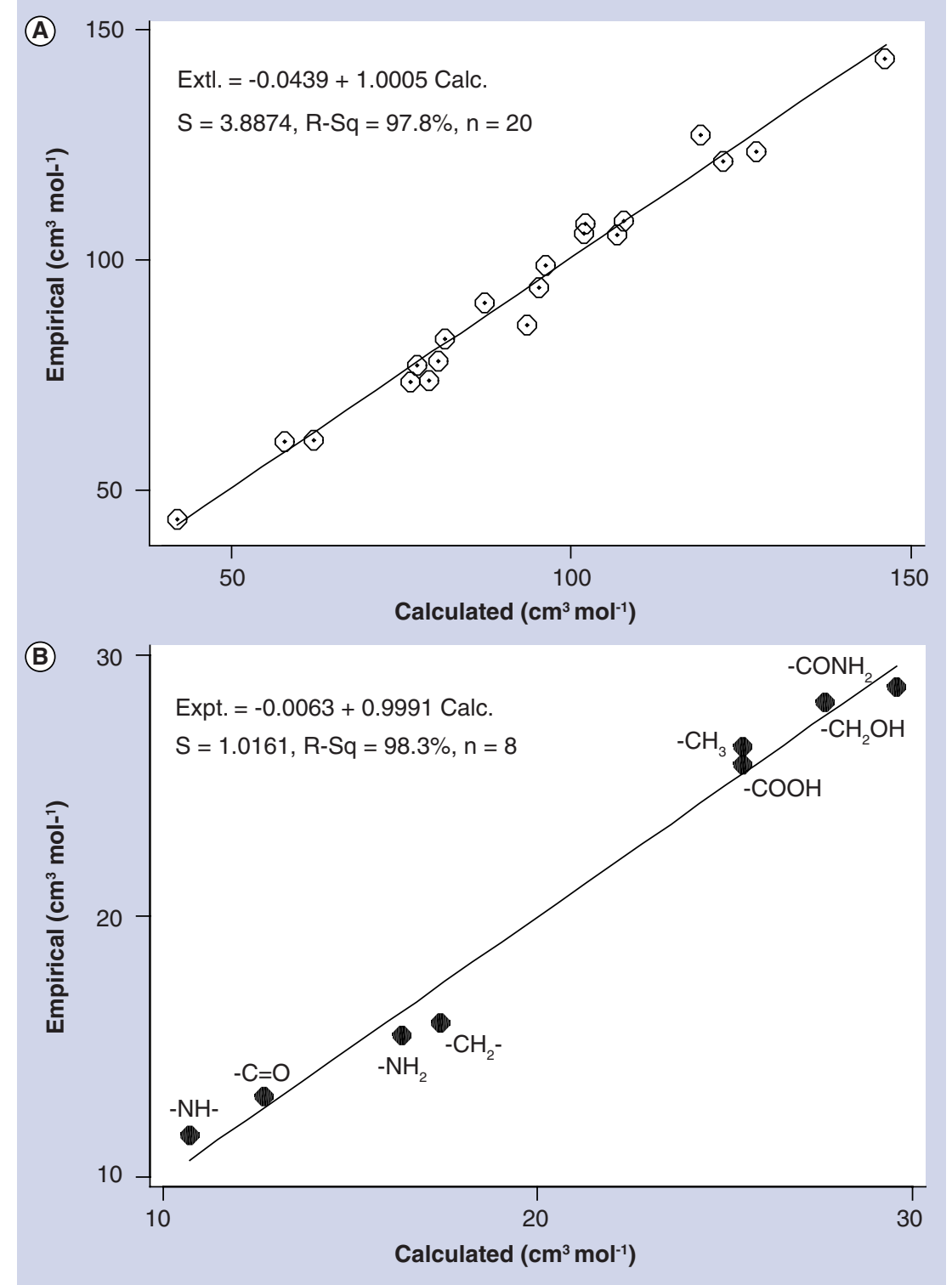

Figure 7. (A) Correlation of the calculated and experimental partial molar volumes of the 20 naturally occurring amino acids. (B) Correlation of the calculated and experimental partial molar volumes of the groups composing the amino acids.

Reproduced with permission from [59]. (C) Wiley-Liss.

When the partition coefficient is expressed as a ratio of molar fractions in the two solvents $s_{1}$ and $s_{2}$, it is labeled with a prime $\left(P^{\prime}\right)$. This partition coefficient is related to the standard free energy of transfer of the solute from one of the two solvent to the other by [62]:

$$
\Delta G_{s_{1} \rightarrow s_{2}}^{0}=R T \ln P^{\prime}
$$

EQuation 47

By virtue of Equation 47, measuring the partition coefficient $P^{\prime}$ is tantamount of measuring the free energy of transfer $\left(\Delta G_{s_{1} \rightarrow s_{2}}^{0}\right)$, and vice versa. 
Table 3. Experimental and calculated partial molar volumes of nucleic acid bases, experimental partial molar volumes of nucleosides, and estimates of the contribution of the ribose sugar to the partial molar volume of the nucleosides.

\begin{tabular}{|c|c|c|c|c|}
\hline Molecule & $\begin{array}{l}V^{\circ}\left(18^{\circ} \mathrm{C}\right) \\
\text { (experimental) }\end{array}$ & $\begin{array}{l}V^{\circ}\left(18^{\circ} \mathrm{C}\right) \\
\text { (calc) }\end{array}$ & $\begin{array}{l}V^{\circ}\left(55^{\circ} \mathrm{C}\right) \\
\text { (experimental) }\end{array}$ & $\begin{array}{l}V^{\circ}\left(55^{\circ} \mathrm{C}\right) \\
\text { (calc) }\end{array}$ \\
\hline \multicolumn{5}{|l|}{ Experimental $^{+}$} \\
\hline \multicolumn{5}{|l|}{ Free bases } \\
\hline Uracil & $70.2 \pm 0.4$ & 69.1 & $74.6 \pm 0.5$ & 74.7 \\
\hline Cytosine & $72.4 \pm 0.4$ & 74.0 & $75.8 \pm 0.5$ & 79.1 \\
\hline Thymine & $86.3 \pm 0.4$ & 86.0 & $91.6 \pm 0.5$ & 90.3 \\
\hline Adenine & $88.0 \pm 0.4$ & 87.5 & $93.5 \pm 0.5$ & 92.2 \\
\hline Guanine $^{\ddagger}$ & $91.6 \pm 1.0^{\ddagger}$ & 91.8 & $97.5 \pm 1.2^{\ddagger}$ & 96.6 \\
\hline \multicolumn{5}{|l|}{ Nucleosides } \\
\hline Uridine & $150.7 \pm 0.6$ & & $154.8 \pm 0.7$ & \\
\hline Cytidine & $152.2 \pm 0.6$ & & $156.4 \pm 0.7$ & \\
\hline Thymidine & $166.4 \pm 0.6$ & & $170.4 \pm 0.7$ & \\
\hline Adenosine & $169.2 \pm 0.6$ & & $175.3 \pm 0.7$ & \\
\hline Guanosine & $172.0 \pm 0.6$ & & $177.8 \pm 0.7$ & \\
\hline \multicolumn{5}{|l|}{ Differences $^{\S}$} \\
\hline Sugar (uracil) & $80.5 \pm 1.0$ & & $80.2 \pm 1.0$ & \\
\hline Sugar (cytosine) & $79.8 \pm 1.0$ & & $80.6 \pm 1.0$ & \\
\hline Sugar (thymine) & $80.1 \pm 1.0$ & & $78.8 \pm 1.0$ & \\
\hline Sugar (adenine) & $81.2 \pm 1.0$ & & $81.8 \pm 1.0$ & \\
\hline Sugar (average) & $80.4 \pm 1.0$ & & $80.4 \pm 1.0$ & \\
\hline \multicolumn{5}{|c|}{$\begin{array}{l}{ }^{\dagger} \text { Experimental data, except for guanine, are obtained from [61]. } \\
\text { "Calculated from EQUATION } \mathbf{4 0 .} \\
{ }^{8} \text { Calculated from EQUATION } \mathbf{3 9} . \\
\text { Reproduced with permission from [60]. () Wiley-VCH. }\end{array}$} \\
\hline
\end{tabular}

The free energies of transfer from cyclohexane to water and from octanol to water of the amino acid side-chains capped with a hydrogen atom (instead of the common $-\mathrm{C}_{\alpha} \mathrm{H}_{\alpha}\left(\mathrm{NH}_{2}\right)$ $\mathrm{COOH}$ group) has been accurately determined by Radzicka and Wolfenden [67]. The reported $\Delta G_{\text {cyclobexane } \rightarrow \text { water }}^{0}$ and $\Delta G_{\text {octanol } \rightarrow \text { water }}^{0}$ constitute an excellent test case for the modeling of the partition coefficient since the side chains of the amino acids constitute a heterogenous molecular set with minimal similarity between its members. Radzicka and Wolfenden report 19 and 17 data points for cyclohexane-water and for octanol-water partitioning, respectively.

The $\Delta G_{\Omega}^{0} \rightarrow_{s 2}$ is modeled in terms of an electrostatic term, namely the side chain CSI of the side chain $\mathrm{CSI}_{R}$, and a measure of bulk, that is, the van der Waals' volume $V(\mathrm{vdW})_{R}$. With these two predictors, the following regression equations are obtained:

$\Delta G_{\text {cyclohexane } \rightarrow \text { water }}^{0}=0.98-1.99 \mathrm{CSI}_{R}+0.00561 V(\mathrm{vdW})_{R}$ $\left[r^{2}=0.887, s=1.924, n=19\right]$ and

$\Delta G_{\text {octanol } \rightarrow \text { water }}^{0}=0.069-0.322 \mathrm{CSI}_{R}+0.00264 V(\mathrm{vdW})_{R}$ $\left[r^{2}=0.759, s=0.566, n=17\right]$

EQUATION 49

where energies are in $\mathrm{kcal} / \mathrm{mol}$, and CSI values and volumes are in atomic units. The correlations between the observed and calculated $\Delta G_{\Omega}^{0} \rightarrow \Omega$ values for the above two cases are graphically displayed in Figure 9.

The linear correlation in EQUATION 48 is stronger than in Equation 49, even though the standard error of the former is close to $2 \mathrm{kcal} / \mathrm{mol}$ (instead of only $\sim 0.6 \mathrm{kcal} / \mathrm{mol}$ for the latter). This can be ascribed to the much wider range of $\Delta G_{\text {cyclohexane-water }}^{0}$ values (approximately from -15 to $+5 \mathrm{kcal} / \mathrm{mol}$ ) compared with the much narrower range of approximately $-1.3-2.5 \mathrm{kcal} / \mathrm{mol}$ in the case of $\Delta G_{\text {octanol-water }}^{0}$. In both regression equations, the polarity index $\left(\mathrm{CSI}_{R}\right)$ enters with a negative coefficient, that is, favoring the transfer to the aqueous phase. In contrast, the measure of bulk, $V(\mathrm{vdW})_{R}$, contributes positively and therefore promotes the transfer to the hydrophobic 
phase. These signs are to be expected since the more polar a molecule, the more it will prefer the aqueous phase and the bulkier the molecule, the less it will favor the transfer to water as the enthalpic cost of creating the cavity and breaking hydrogen bonds appears to overwhelm a possible entropic gain.

Finally, the smaller magnitude of the two coefficients in Equation 49 with respect to EQUATION 48 is consistent with a more significant environmental change experienced by the solute upon its transfer from cyclohexane to water (nonpolar to highly polar), as opposed to when it leaves octanol to water (polar to highly polar).

There are several other correlations that were found between calculated properties of the amino acid side-chains such as $\operatorname{CSI}_{R}$ and $V(\mathrm{vdW})_{R}$, including the accurate predictions of the Gibbs energy of transfer from the vapor phase to aqueous solution, the change in the stability of proteins upon single-point mutations (including both the enthalpic and entropic contributions), and some striking regularities in the genetic code itself $[59,60]$.

\section{- Transferable atom equivalent}

Breneman introduced the concept of 'transferable atom equivalent' (TAE) [68-71] in an effort to obtain $a b$ initio-quality results bypassing the notorious $N^{n}$ computational scaling of these calculations (generally, $2 \leq n \leq 5$, for HartreeFock $<$ DFT, and commonly used higher levels of theory, where $N$ is the size of the basis set).

A TAE is a mononuclear atomic region of space filled with electron density delimited by zero-flux surfaces in the gradient vector field of the electron density extracted from a parent molecule at its zero-flux surface. Extracted atoms representing a large number of differing combinations of elements, atom types, and immediate electronic environments, are stored in a computerized database. A program called RECON is then used to assemble the electron densities (and other properties) of a target large molecule by matching the appropriate zero-flux surfaces of different TAEs recalled from the database.

Once a TAE fragment is recalled, the fragment is rotated to minimize the mismatch of its surface to the growing end of the reconstructed molecule. The TAE-RECON theoretical reconstruction then proceeds by an automatic melding of the nearly matching zero-flux surfaces. The quantum stress tensor is allowed to exert pressure on the two surfaces being melded until the equalization of the pressure is achieved

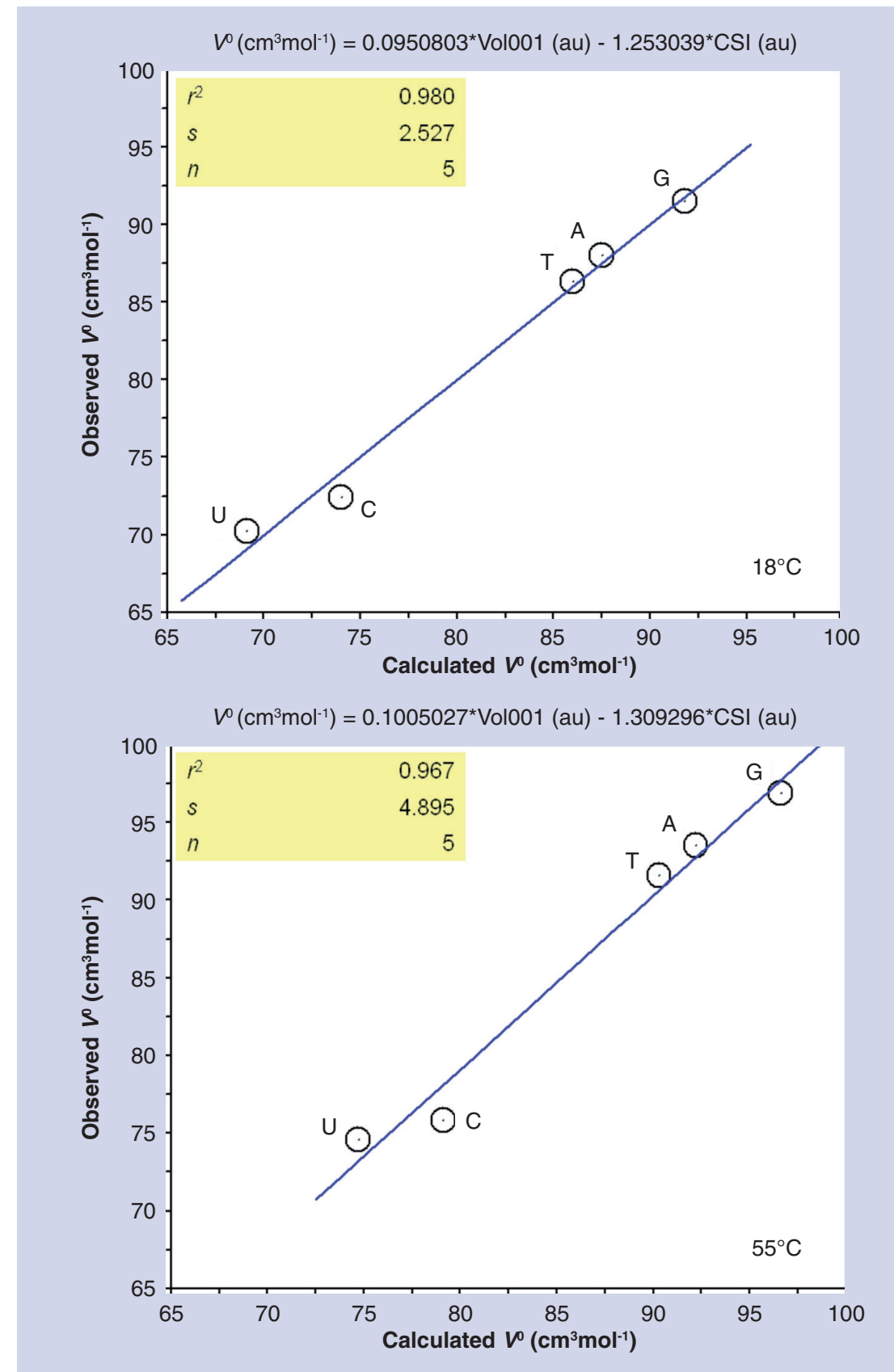

Figure 8. Correlation of calculated and predicted aqueous partial molar volumes at infinite dilution of the five nucleic acid bases adenine $(A)$, guanine (G), cytosine (C), thymine $(\mathrm{T})$ and uracil $(\mathrm{U})$ at $18^{\circ} \mathrm{C}$ and $55^{\circ} \mathrm{C}$. Reproduced with permission from [60]. (C) Wiley-VCH.

on both sides. At this point, the two nearly matched surfaces have merged into one resultant surface of zero-flux. The TAE-RECON melding of interatomic surfaces is accompanied by an adjustment of the bond length between the two newly bonded atoms. Similar sequential addition of new TAEs is repeated until the molecule is synthesized from the database of atomic fragments. 

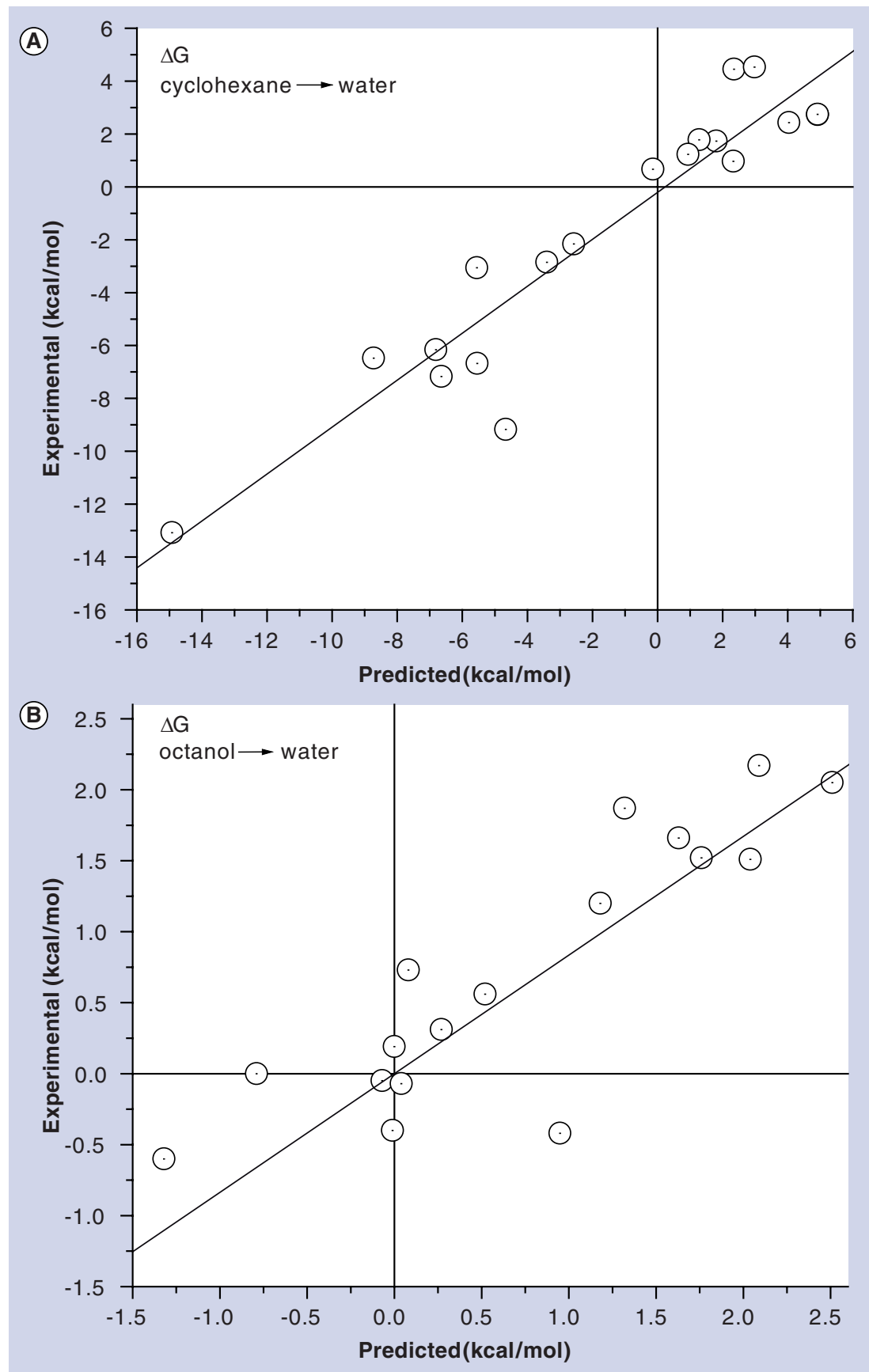

Figure 9. (A) Experimental and calculated standard Gibbs energies of transfer of 19 amino acid side chains from cyclohexane to water and (B) of 17 amino acid side chains from octanol to water. The side chains are capped with a hydrogen atom in all cases, the hydrogen atom replacing the common $-\mathrm{C}_{\alpha} \mathrm{H}_{\alpha}\left(\mathrm{NH}_{2}\right) \mathrm{COOH}$ group.
The record that stores the information on a given TAE in the library includes the derivatives of atomic properties with respect to small radial variations in each unique position of the zeroflux surface of the TAE. The derivatives are used to correct for the changes in the atomic properties resulting from the change in the interatomic surface in the melding procedure.
Once the target molecule is reconstructed by the automated melding of TAEs, the molecular descriptors are then calculated by simple or vectorial sums of the properties of the composing TAEs. The reconstructed descriptors include, for example, the total molecular energy, the total molecular volume, the surface area defined by a given isodensity envelope, the electrostatic potential, the molecular dipole moment, the Fukui function and the bare nuclear potential. (A relatively recent and comprehensive review by Sukumar and Brenemann provides several technical details on how this is achieved in practice [71]).

The TAE approach has been shown to be of great value and potential in QSAR/QSPR studies [70]. For example, the acute toxicity of a large dataset of organic molecules to fish is predicted accurately by TAE-RECON reconstructed molecular descriptors. In this study, the original 375 organic molecule dataset has been divided into a training set of $300 \mathrm{~mol}$ ecules and a test set of 75 molecules. The prediction on the test set exhibited a 'leave one out' $q^{2}=0.81$ [71]. The significance of these results is the speed with which $a b$ initio-quality electron density and descriptors/properties of large molecular sets can be obtained in the modeling of their pharmacological properties.

\section{Use of QTAIM delocalization indices to predict NMR JJ coupling constants}

Nuclear magnetic resonance is an essential tool in the arsenal of the medicinal chemist. Here, we show how the delocalization index, defined by Equations 12 \& 13, can be used in the empirical prediction of NMR $J J$ couplings between pairs of protons and/or pairs of fluorine nuclei in a molecule. This can be of help in the interpretation and peak assignment of complex NMR spectra.

It is a matter of routine to calculate NMR shielding and coupling constant data in standard computational chemistry packages such as Gaussian [32] or GAMESS [33] so a reader may wonder, justifiably, about the motivation to resort to empiricism in the prediction of $J J$ coupling data. The reason is to provide further support for the physical nature of the atomic properties calculated within QTAIM.

In this case, the tested property is the delocalization index, which is an example of a twoelectron property of two atoms in a molecule (in contrast to a one-electron property of one atom in the molecule such as the atomic charge, 
for example). Since the use of electron densityderived properties in QSAR/QSPR is in its infancy, every test of this emerging technology contributes to the confidence we have in its application to drug design.

A principal mechanism by which spin information is transmitted between two nuclei of half-integral spin (e.g., ${ }^{1} \mathrm{H},{ }^{13} \mathrm{C},{ }^{19} \mathrm{~F}$ ) in a molecule is the so-called Fermi contact term. This term is generally the lead one that determines the strength of J-coupling (or scalar spin coupling) [72-74]. The coupling constant may have positive or negative values depending on the relative orientation of the spin states involved in the absorption of electromagnetic energy.

The prediction of $J$-coupling constants requires very costly calculations since very large basis sets are necessary for an accurate representation of the electron density at or near the atomic nuclei [75-77]. It has been shown that an alternative empirical model that bypasses the requirement of extremely accurate representation near the nuclei can be achieved within the QTAIM framework by the use of the delocalization indices $\delta(A, B)$ [78-80]. Here, we only highlight the predictive value of this modeling, the reader can consult the original literature for a deeper discussion of the theoretical foundations of this modeling.

The Fermi contact contribution to the coupling constant between two nuclei $J_{\mathrm{nn}}$, is related to the electronic exchange between the positions of their nuclei [81]:

$$
J_{n n^{\prime}} \propto \delta(A, B)
$$

\section{Equation 53}

Experimental proton-proton $\left(\mathrm{H}, \mathrm{H}^{\prime}\right)$ coupling constants in polycyclic aromatic hydrocarbons $J_{\mathrm{HH}^{\prime}}$ have indeed been found to be strongly, linearly correlated to the theoretically calculated values of $\delta\left(\mathrm{H}, \mathrm{H}^{\prime}\right)$ as predicted from the assumptions previously discussed leading to Equation 53. Experimental coupling constants in Hertz and $\delta\left(\mathrm{H}, \mathrm{H}^{\prime}\right)$ fit the following regression equation [78]:

$$
\begin{aligned}
& J_{\mathrm{HH}^{\prime}}=-0.1369+1274.9 \delta\left(\mathrm{H}, \mathrm{H}^{\prime}\right) \\
& {\left[r^{2}=0.989, s=0.375, n=29\right]}
\end{aligned}
$$

EQUATION 54

In a follow-up study, the fluorine-fluorine $\left(\mathrm{F}, \mathrm{F}^{\prime}\right)$ coupling constants in doubly fluorinated aromatic hydrocarbons, has also been shown to be linearly strongly correlated to $\delta\left(\mathrm{F}, \mathrm{F}^{\prime}\right)$ by [80]:

$$
\begin{aligned}
& J_{\mathrm{FF}}=3.696+959.953 \delta\left(\mathrm{F}, \mathrm{F}^{\prime}\right) \\
& {\left[r^{2}=0.85, s=10.38, n=33\right]}
\end{aligned}
$$

EQUATION 55

where $J_{\mathrm{FF}}$ is in $\mathrm{Hz}$.

The linear correlations between coupling constants and delocalization indices expressed in Equations 54 \& 55 are displayed in Figure 10. The correlation of the proton-proton coupling constant with the delocalization index is stronger than the corresponding correlation in the case of the fluorine atoms.

$$
J_{n n}=\underbrace{-\frac{2}{3 h}\left[\frac{16 \pi \beta \hbar}{3}\right]^{2} \gamma_{n} \gamma_{n} \frac{1}{\Delta E}}_{\text {constant }} \sum_{i} \sum_{j}\left\langle\phi_{i}(\mathbf{n}) \delta\left(\mathbf{n}_{n}\right) \phi_{j}(\mathbf{n})\right\rangle\left\langle\phi_{j}\left(\mathbf{r}_{2}\right) \delta\left(\mathbf{r}_{2 n}\right) \phi_{i}\left(\mathbf{r}_{2}\right)\right\rangle
$$

\section{EOUATION $\mathbf{5 0}$}

in standard notation, where $\delta\left(\mathbf{r}_{k n}\right)$ is a Dirac $\delta$ function that selects the value of the integral at the position of nucleus $n\left(\mathbf{r}_{k n}=0\right)$. It was conjectured that if the density at a nucleus is, on average, proportional to the density within the entire basin of that atom, then [78]:

$$
\begin{aligned}
& \left\langle\phi_{i}\left(\mathbf{r}_{1}\right) \delta\left(\mathbf{r}_{1 n}\right) \phi_{j}\left(\mathbf{r}_{1}\right)\right\rangle\left\langle\phi_{j}\left(\mathbf{r}_{2}\right) \delta\left(\mathbf{r}_{2 n}\right) \phi_{i}\left(\mathbf{r}_{2}\right)\right\rangle \underset{\sim}{\propto} \\
& \left\langle\phi_{i}\left(\mathbf{r}_{1}\right) \phi_{j}\left(\mathbf{n}_{1}\right)\right\rangle_{\mathrm{F}}\left\langle\phi_{j}\left(\mathbf{r}_{2}\right) \phi_{i}\left(\mathbf{r}_{2}\right)\right\rangle_{\mathrm{F}} \equiv S_{j i}(A) S_{i j}(B)
\end{aligned}
$$

Equation 5 I

which, when inserted into the double sum, yields:

$$
J_{n n} \propto \sum_{i} \sum_{j} S_{j i}(A) S_{i j}(B)
$$

EQUATION 52

or
This can be ascribed to the greater flexibility of the fluorine atom to distort and, hence, to further depart from the approximate sphericity that constitutes the crucial assumption behind the postulated proportionality in EQUATION $5 \mathbf{I}$.

\section{MEP \& bioisosteric similarity}

The MEP has long been used in medicinal chemistry and this section cannot review this massive literature (for a necessarily incomplete sampling of the literature the reader is referred to [14-16,68,82-92] and the literature cited therin). Instead, we review a very specific recent application where the MEP has been shown to uncover the basis of bioisosteric similarity of electron density fragments within a molecule. In this study, the bioisosteric similarity of 


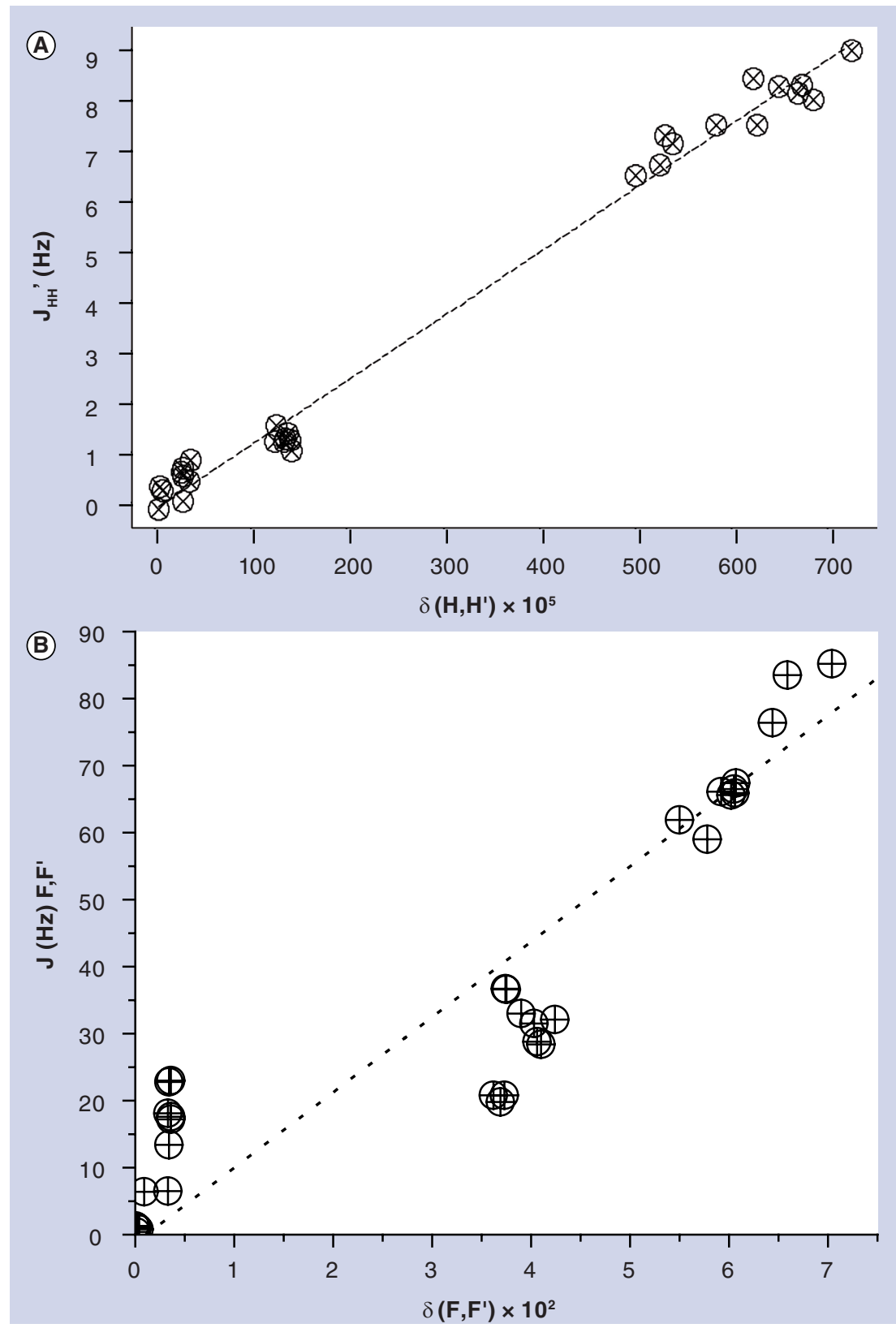

Figure 10. (A) Display of the linear correlation between the $\mathrm{H}-\mathrm{H}^{\prime}$ delocalization index, $\delta\left(H, H^{\prime}\right)$, and the experimental proton-proton NMR spin-spin coupling in $\mathrm{Hz}$ (cycles/sec) obtained from a set of small polycyclic aromatic hydrocarbons. (B) A display of the linear correlation between the $F-F^{\prime}$ delocalization index, $\delta\left(F_{,} F^{\prime}\right)$, and the experimental fluorine-fluorine NMR spin-spin coupling in $\mathrm{Hz}$ obtained from a set of difluorinated polycyclic aromatic hydrocarbons.

(A) Adapted with permission from [78]. (C) American Chemical Society.

(B) Data from [80]. question of how they behave similarly at the level of drug-receptor interaction. The structures in Figure II do not provide an obvious path for predicting their empirically established bioisosteric similarity [93-97].

The electrostatic potential (ESP) generated by tetrazole and carboxylate has, recently, been shown to exhibit an identical topology and a very similar topography. In both cases, the ESP is remarkably similar whereby it is characterized by the disposition of four coplanar local minima at positions consistent with lone pairs [17] (Figure 12). Furthermore, the geometrical disposition of these four regions of locally minimum ESP are nearly identical in the two bioisosters. This geometrical arrangement is not significantly altered by the nature of the capping group, be it methyl as in Figures II \& I2, or a chlorine atom, or a hydrogen atom [17]. This similarity of the ESP cannot be easily anticipated on the basis of the chemical structures in Figure II.

It is also found that the average electron density of the bioisosteric groups is identical, which is significant as even the same group in different environments generally exhibits significantly different average electron densities [17].

The similarity of the ESP suggests a model for the receptor region that binds to the two bioisosteres in an electrostatic 'lock-and-key' fashion. It is hypothesized that four positively charged regions in the receptor are spatially oriented so as to maximize their interactions with the four lobes of maximally negative ESP generated by the charge distribution of the bioisosteres.

\section{Conclusion}

This review has, hopefully, demonstrated that questions of medicinal chemistry can be meaningfully formulated in terms of scalar fields, namely, the electron (and/or total charge) density and its associated ESP/MEP. Besides their fundamental nature as sources for all molecular properties, both the electron density and the MEP can also be experimentally determined from x-ray diffraction data $[30,31]$, as discussed above.

The reliance on observable physical fields to model biological activity (or properties directly related to biological activity such as $\log P$ ) removes a major source of uncertainty in the modeling when compared with models constructed on the basis of ad hoc descriptors. The use of the quantum theory of atoms in molecules 
and of MEPs to generate molecular descriptors based on physical fields is still in its infancy but its potential utility is undeniably considerable.

\section{Future perspective}

The importance of the electron density came to the fore in the early 1960s through the groundbreaking work of scientists such as Kohn $[18,98]$ and Bader [99-102] and their co-workers.

Kohn and his school laid the foundations of modern DFT, and showed that the electron density is a basic variable that is functionally related to all the properties of the ground state. DFT has now become one of the most widely used method in theoretical chemistry with applications ranging from solid state, to biochemistry, and medicinal chemistry.

In the meantime, Bader recognized the fundamental role of the electron density in the theory of chemistry and was the first to recognize the relation between the transferability of all properties of a molecular fragment and the corresponding transferability (or similarity) of the underlying electron density. Bader and coworkers extended quantum mechanics itself to proper open system (atoms in molecules) after it has long been thought to apply only to total systems (since one cannot partition the hamiltonian, for example). QTAIM, benefiting from four decades of development, has now reached a stage of maturity and elegance that has extended its application to many branches of theoretical chemistry where it is used to extract chemical information and make predictions about chemical systems.

Both of these two lines of research, therefore, complement each another: DFT is used to calculate the density and properties of entire molecules as closed (whole) quantum systems; QTAIM tells us how to uniquely partition the system into proper open quantum subsystems to recover the properties of the atoms in the molecule or crystal. While complementary to one another, these two branches of theoretical chemistry have evolved each into its own coherent intellectual and mathematical structure, but quite independent from one another until the 1990s.

The 1990s witnessed an explosive growth in computer powers and algorithmic developments epitomized by the recent versions of software such as Gaussian XX [32] and GAMESS [33] (for nonperiodic quantum calculations) and CRYSTAL [103] (for periodic calculations). The inclusion of popular DFT functionals such as

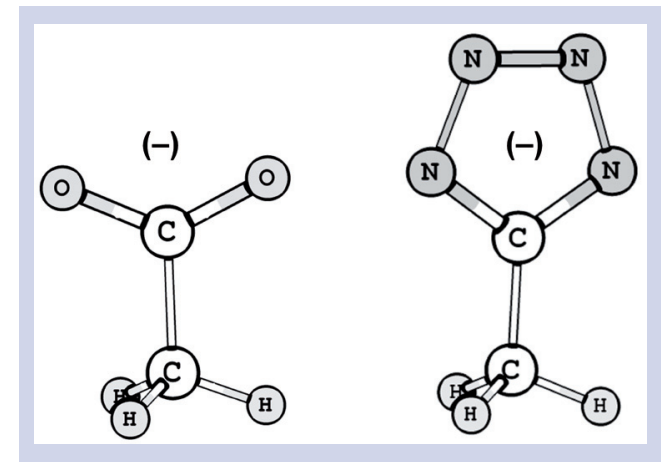

Figure 11. Representation of actetate and of 5-methyl tetrazole anion in their gas-phase optimized geometries.

the B3LYP $[104,105]$ in these software was accompanied by an dramatic increase in the usage of DFT by groups from all walks of research, notably from the biological and medicinal chemistry arenas [11,106-112]. These developments have been echoed by the availability of electrondensity analysis software, most prominently, AIMPAC of Bader's group [113,201], MORPHY of Popelier [114,115], Biegler's AIM2000 [202,116,117], and more recently the very powerful and comprehensive program developed by Todd A. Keith called AIMALL [118]. All these codes analyze the densities and wave functions of nonperiodic system obtained from quantum mechanical calculation. Gatti developed an interface for the CRYSTAL code called Topond, which analyses densities obtained from periodic calculations [119].

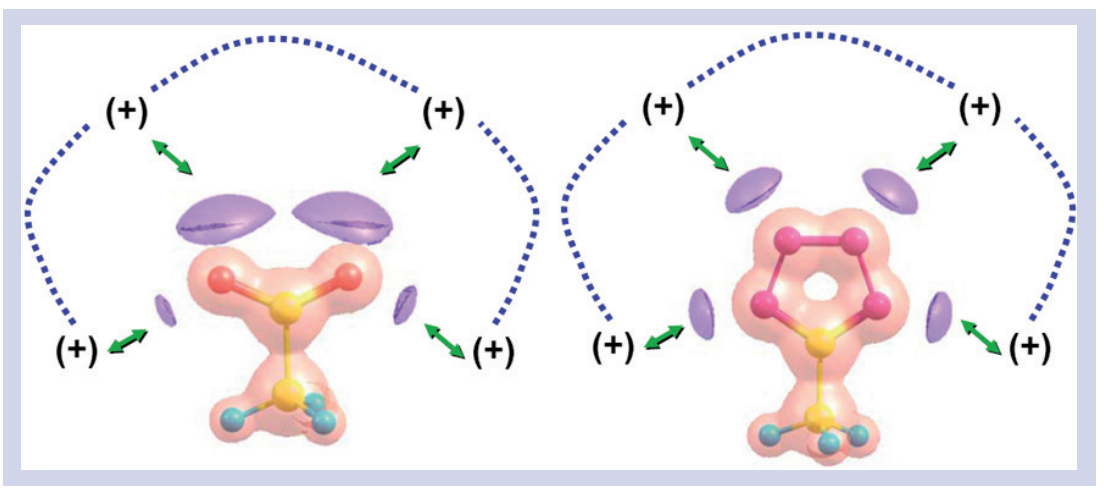

Figure 12. Electrostatic potential around the two bioisosteres actetate and of 5-methyl tetrazole anion along with a postulated model of a common region of a hypothetical receptor on the basis of 'lock-and-key' electrostatic complementarity. Violet isosurfaces enclose negative values of the molecular electrostatic potential and pink envelopes enclose positive molecular electrostatic potential regions. The postulated complementary region of the receptor bears four positive charges oriented to oppose the four negative local minima in the electrostatic potential of the two bioisosteres.

Reproduced with permission from [17]. (C) Elsevier. 
Non-less spectacular advances in speed and accuracy of x-ray diffraction techniques have been achieved at the same time thanks to the invention of the CDD camera, the increasing accessibility to bright synchrotron sources, and major algorithmic and software improvements for the refinement of the diffraction data. As a result, the determination of very accurate electron density maps from $\mathrm{x}$-ray crystallography has become accessible to numerous research laboratories in the world. QTAIM, having its starting point the electron density, has quickly acquired the status of the single most important theory used to analyze experimental density maps nowadays [30,31,120-127]. Experimental densities are currently analyzed by several software, we mention among them XD [128] and MOPRO [129,130] as examples.

In the recent past, the analysis of the electron density through QTAIM has been applied to predict and interpret molecular properties at the atomic level in a vastly diverse range of research. Examples of application include the prediction and understanding of heats of formation [131], magnetic susceptibilities [132,133], atomic electrostatic moments and polarizabilities
[134,135], Raman intensities [134-137], IR intensities [138,139], electron localization and delocalization $[140,141], p K_{a}$ [55], biological and physicochemical properties of the amino acids [59,142-145], protein retention times [146], HPLC column capacity factors [70], NMR spin-spin coupling constants $[78,80]$, atom typing in force fields design for proteins [147], pharmacophore/active site searching $[47-49,51,148]$ the use of transferable fragments in the reconstruction of large biomolecules not amenable to direct computation $[28,69,149]$ or crystallization [125].

Given such an extremely wide range of applications, it appears to be a matter of time before QTAIM will play an important role as a generator of high-quality and physically meaningful descriptors for QSAR/QSPR modeling in drug and materials research. The great Niels Bohr attributably replied, "it is exceedingly difficult to make predictions, particularly about the future" when asked to "predict the influence Quantum Physics will have on the world in the future", because the present state of a system cannot be known with infinite precision, let alone its evolution in the future. So we make no predictions, but rather a recommendation.

Executive summary

- Molecular similarity is an elusive concept that can be discussed and quantified satisfactorily from a study of the topology of the molecular electron density.

- The concepts of similarity and transferability of an atom or a group of atoms from one molecular environment to another are related and can be quantified through the definition of atomic and group contributions to the corresponding molecular properties. This additivity applies to scalar fields (such as the electron density, the various energy densities and the volume), vectors (e.g., dipole moment), or tensor properties (e.g., electrostatic quadrupole moment tensor, or polarizability [which is also an example of a responseproperty]). This is achieved through the analysis of molecular electron densities provided within the framework of the quantum theory of atoms in molecules (QTAIM).

- Atoms in a molecule are true open systems that communicate with one another. The delocalization of electrons between any two given atoms in a molecule, bonded or not, is quantified within QTAIM by the so-called delocalization index. This index is an excellent predictor of NMR JJ coupling constants. This property is listed among those well predicted by QTAIM since its application in quantitative structure-activity relationships/quantitative structure-property relationships (QSAR/QSPR) is still in its infancy and the more examples the more our confidence in its general applicability to QSAR/QSPR-type studies.

- QTAIM defines bond properties and atomic/group properties. Both of these two categories of QTAIM properties have been used as descriptors in the construction of QSAR/QSPR models.

- Bond properties are shown to be very useful in the prediction of properties such as $p K_{a}$ of very different molecular sets. Bond properties have even been used in the automatic searching of pharmacophores through an approach pioneered by Popelier and termed quantum molecular similarity.

- Atomic properties have been shown to be excellent predictors of $p K_{a^{\prime}}$ partial molar volumes, partition coefficient, to mention a few recent examples.

- The molecular electrostatic potential (MEP) arises from the total charge density (the discrete distribution of point-like charges at the positions of the nuclei in addition to the diffuse, continuous, electron density). The MEP is a fundamental molecular descriptor that has had an immense range of applicability in the design of drugs. In this review, we limit ourselves to only one recent application since it was within the context of a QTAIM study. The so-far unexplained bioisosteric similarity of the tetrazole and acetate anions has been rationalized, in this study, on the basis of the very similar topologies of the MEPs of these two bioisosteres.

- The use of QTAIM in QSAR/QSPR studies, and in medicinal chemistry in general, is still at a very early stage, but it is a promising line of research. 


\section{Acknowledgements}

Professor Keneth Adam, Professor Paul LA Popelier, the American Chemical Society, Elsevier, Wiley-Liss, and Wiley-VCH, are acknowledged for their kind permissions to reproduce copyrighted materials. $\mathrm{Dr}$ Todd A Keith is thanked for supplying a copy of AIMALL used to generate some of the data reported in the literature reviewed in this work.

\section{Financial \& competing interests disclosure}

Grant support of The Natural Sciences and Engineering Research Council of Canada, Canada Foundation for Innovation, The Killam Trusts, and Mount Saint Vincent University is gratefully acknowledged. The authors have no other relevant affiliations or financial involvement with any organization or entity with a financial interest in or financial conflict with the subject matter or materials discussed in the manuscript apart from those disclosed.

No writing assistance was utilized in the production of this manuscript.

\section{Bibliography}

1 Carbó R, Leyda L, Arnau M. How similar is a molecule to another? An electron density measure of similarity between two molecular structures. Int. J. Quantum Chem. 17, 1185-1189 (1980).

2 Carbó-Dorca R, Mezey PGE. Advances in Molecular Similarity, Vol. 1. Jai Press Inc, London, UK (1996).

3 Carbó-Dorca R, Mezey PGE. Advances in Molecular Similarity, Vol. 2. Jai Press Inc, London, UK (1998).

4 Carbó-Dorca R, Robert D, Amat LL, Gironé X, Besalú E. Molecular Quantum Similarity in QSAR and Drug Design. Springer: Berlin, Germany (2000).

5 Johnson MA, Maggiora GM (Eds). Concepts and Applications of Molecular Similarity. John Wiley and Sons, Inc., NY, USA (1990).

6 Bader RFW, Popelier PLA, Chang C. Similarity and complementarity in chemistry. J. Mol. Struct. (Theochem), 255, 145-171 (1992).

7 Attwood TK, Parry-Smith DJ. Introduction to Bioinformatics. Prentice Hall, London, UK (1999).

8 Gibas C, Jambeck P. Developing Bioinformatics Computer Skills. O'Reilly: Cambridge, UK (2001).

9 Mount DW. Bioinformatics: Sequence and Genome Analysis (Second Edition). Cold Spring Harbor Laboratory Press, New York, USA (2004).
10 Janezic D, Milicevic A, Nikolic S, Trinajstic N. Graph Theoretical Matrices in Chemistry (Mathematical Chemistry Monographs, Vol. 3). University of Kragujevac, Kragujevac, Serbia (2007).

11 Doucet JP, Weber J. Computer-Aided Molecular Design: Theory and Applications. Academic Press Ltd, London, UK (1996).

12 Gadre SR, Kulkarni SA, Suresh CH, Shrivastava IH. Basis set dependence of molecular electrostatic potential topography: a case study of substituted benzenes. Chem. Phys. Lett. 239, 273-281 (1995).

13 Suresh CH, Gadre SR. Novel electrostatic approach to substituent constants: doubly substituted benzenes. J. Am. Chem. Soc. 120, 7049-7055 (1998).

14 Gadre SR. Topography of atomic and molecular scalar fields. In: Computational Chemistry: Reviews of Current Trends. World Scientific, Singapore 1-53 (1999).

15 Gadre SR, Shirsat RN. Electrostatics of Atoms and Molecules. Universities Press, Hyderabad, India (2000).

16 Roy D, Balanarayan P, Gadre SR. An appraisal of Poincaré-Hopf relation and application to topography of molecular electrostatic potentials. J. Chem. Phys. 129, 174103 (2008).

17 Matta CF, Arabi AA, Weaver DF. The bioisosteric similarity of the tetrazole and carboxylate anions: clues from the topologies of the electrostatic potential and of the electron density. Eur. J. Med. Chem. 45, 1868-1872 (2010).

18 Hohenberg P, Kohn W. Inhomogeneous electron gas. Phys. Rev. B136, 864-871 (1964).

19 Riess I, Münch W. The theorem of Hohenberg and Kohn for subdomains of a quantum system. Theor. Chim. Acta. 58, 295-300 (1981).

20 Bader RFW, Becker P. Transferability of atomic properties and the theorem of Hohenberg and Kohn. Chem. Phys. Lett. 148, 452-458 (1988).

21 Mezey PG. The holographic electron density theorem and quantum similarity measures. Mol. Phys. 96, 169-178 (1999).

22 Constans P, Amat L, Carbó-Dorca R. Toward a global maximization of the molecular similarity function: superposition of two molecules. J. Comput. Chem. 18, 826-846 (1997).

23 McMahon AJ, King PM. Optimization of Carbó molecular similarity index using gradient methods. J. Comput. Chem. 18, 151-158 (1997).
24 Bader RFW. Encyclopedia of Computational Chemistry. Schleyer PvR (Ed.). John Wiley and Sons, Chichester, UK 64-86 (1998).

25 Bader RFW. A quantum theory of molecular structure and its applications. Chem. Rev.91, 893-928 (1991).

26 Bader RFW. Atoms in Molecules: a Quantum Theory. Oxford University Press, Oxford, UK (1990).

27 Popelier PLA. Atoms in Molecules: An Introduction. Prentice Hall, London, UK (2000).

28 Bader RFW, Matta CF, Martín FJ. Atoms in medicinal chemistry. Medicinal Quantum Chemistry. Wiley-VCH, Weinheim, Germany 201-231 (2003).

29 Matta CF, Boyd RJ (Eds). The Quantum Theory of Atoms in Molecules: from Solid State to DNA and Drug Design. Matta CF, Boyd RJ (Eds). Wiley-VCH, Weinheim, Germany (2007).

30 Coppens P. X-ray Charge Densities and Chemical Bonding. Oxford University Press, Inc, NY, USA (1997).

31 Koritsanszky TS, Coppens P. Chemical applications of x-ray charge-density analysis. Chem. Rev. 101, 1583-1628 (2001).

32 Frisch MJ, Trucks GW, Schlegel HB et al. Gaussian 09, Gaussian Inc., Wallingford, CT, USA (2009).

33 Schmidt MW, Baldridge KK, Boatz JA et al. General atomic and molecular electronicstructure system (GAMESS). J. Comput. Chem. 14, 1347-1363 (1993).

34 Bader RFW. A bond path: a universal indicator of bonded interactions. J. Phys. Chem. A 102, 7314-7323 (1998).

35 Runtz GR, Bader RFW, Messer RR. Definition of bond paths and bond directions in terms of the molecular charge distribution. Can. J. Chem. 55, 3040-3045 (1977).

36 Popelier PLA. Integration of atoms in molecules: a critical examination. Mol. Phys. 87, 1196-1187 (1996).

37 Kosov DS, Popelier PLA. Atomic partitioning of molecular electrostatic potentials. J. Phys. Chem. A 104, 7339-7345 (2000).

38 Kosov DS, Popelier PLA. Convergence of the multipole expansion for electrostatic potentials of finite topological atoms. J. Chem. Phys. 113, 3969-3974 (2000).

39 Popelier PLA, Joubert L, Kosov DS. Convergence of the electrostatic interaction based on topological atoms. J. Phys. Chem. A 105, 8254-8261 (2001).

40 Bader RFW, Nguyen-Dang TT. Quantum theory of atoms in molecules - Dalton revisited. Adv. Quantum Chem. 14, 63-124 (1981). 
41 Bader RFW. Principle of stationary action and the definition of a proper open system. Phys. Rev. B 49, 13348-13356 (1994).

42 Cremer D, Kraka E. Chemical bonds without bonding electron density - does the difference electron-density analysis suffice for a description of the chemical bond? Angew. Chem. Int. Ed. Engl. 23, 627-628 (1984).

43 Fradera X, Austen MA, Bader RFW. The Lewis model and beyond. J. Phys. Chem. A 103, 304-314 (1999).

44 Matta CF, J. Hernández-Trujillo. Bonding in polycyclic aromatic hydrocarbons in terms of the electron density and of electron delocalization. J. Phys. Chem. A 107, 7496-7504 (2003) (Erratum in: J. Phys. Chem A, 109, 10798 [2005]).

45 Zhurova EA, Matta CF, Wu N, Zhurov VV, Pinkerton AA. Experimental and theoretical electron density study of estrone. J. Am. Chem. Soc. 128, 8849-8861 (2006).

46 Hammett LP. Physical Organic Chemistry: Reaction Rates, Equilibria, and Mechanisms (Second Edition). McGraw-Hill Book Company, NY, USA (1970).

47 Popelier PLA. Quantum molecular similarity. 1. BCP space. J. Phys. Chem. A 103, 2883-2890 (1999).

48 O’Brien SE, Popelier PLA. Quantum molecular similarity. Part 2: the relation between properties in BCP space and bond length. Can. J. Chem. 77, 28-36 (1999).

49 O’Brien SE, Popelier PLA. Quantum molecular similarity. 3. QTMS descriptors. J. Chem. Inf. Comput. Sci. 41, 764-775 (2001).

50 O’Brien SE, Popelier PLA. Quantum molecular similarity: use of atoms in molecules derived quantities as QSAR variables. In: European Congress on Computational Methods in Applied Sciences and Engineering (ECCOMAS 2000) Barcelona, Spain (2000).

51 Chaudry UA, Popelier PLA. Estimation of pKa using quantum topological molecular similarity descriptors: application to carboxylic acids, anilines and phenols. J. Org. Chem. 69, 233-241 (2004).

52 Singh N, Loader RJ, O’Malley PJ, Popelier PLA. Computation of relative bond dissociation enthalpies (BDE) of phenolic antioxidants from quantum topological molecular similarity (QTMS). J. Phys. Chem. A 110, 6498-6503 (2006).

53 Hawe GI, Alkorta I, Popelier PLA. Prediction of the basicities of pyridines in the gas phase and in aqueous solution. J. Chem. Inf. Model. 50, 87-96 (2010).
54 Popelier PLA. Developing Quantum Topological Molecular Similarity (QTMS). In: Quantum Biochemistry: Electronic Structure and Biological Activity (Volume 2). Matta CF (Ed.). Wiley-VCH, Weinheim, Germany 669-691 (2010).

55 Adam KR. New density functional and atoms in molecules method of computing relative $\mathrm{p}$ Ka values in solution. J. Phys. Chem. A 106, 11963-11972 (2002).

56 Millero FJ, Surodo AL, Shin C. The apparent molal volumes and adiabatic compressibilities of aqueous amino acids at $25^{\circ} \mathrm{C}$. J. Phys. Chem. 82, 784-792 (1978).

57 Collantes ER, Dunn WJI. Amino acid side chain descriptors for quantitative structureactivity relationship studies of peptide analogues. J. Med. Chem. 38, 2705-2713 (1995).

58 Hinz HJ Thermodynamic Data for Biochemistry and Biotechnology. SpringerVerlag, Berlin, Germany (1986).

59 Matta CF, Bader RFW. Atoms-in-molecules study of the genetically-encoded amino acids. III. Bond and atomic properties and their correlations with experiment including mutation-induced changes in protein stability and genetic coding. Proteins: Struct. Funct. Genet. 52, 360-399 (2003).

60 Matta CF. From atoms in amino acids to the genetic code and protein stability, and backwards. In: Quantum Biochemistry: Electronic Structure and Biological Activity (Volume 1). Matta CF (Ed.). Wiley-VCH: Weinheim 423-472 (2010).

61 Lee A, Chalikian TV. Volumetric characterization of the hydration properties of hetercyclic bases and nucleosides. Biophys. Chem. 92, 209-227 (2001).

62 Leo A, Hansch C, Elkins D. Partition coefficients and their uses. Chem. Rev. 71, 525-616 (1971).

63 Helmer F, Kiehs K, Hansch C. The linear free-energy relationship between partition coefficients and the binding and conformational perturbation of macromolecules by small organic compounds. Biochem. 7, 2858-2863 (1968).

64 Hansch C. A quantitative approach to biochemical structure-activity relationships. Acc. Chem. Res. 2, 232-239 (1969).

65 Tute MS. Principles and practice of Hansch analysis: a guide to structure-activity correlation for the medicinal chemist. In: Advances in Drug Research (Vol. 6). Academic Press, London, UK 1-77 (1971).

66 Patrick GL. An Introduction to Medicinal Chemistry. Oxford University Press, Oxford, UK (2005).
67 Radzicka A, Wolfenden R. Comparing the polarities of the amino acids: side-chain distribution coefficients between the vapor phase, cyclohexane, 1-octanol, and neutral aqueous solutions. Biochem. 27, 1664-1670 (1988).

68 Breneman CM, Weber LW. Transferable atom equivalents. Molecular electrostatic potentials from the electric multipoles of PROAIMS atomic basins. In: NATO ASI Series: The Application of Charge Density Research to Chemistry and Drug Design. Plenum Press, NY, USA 357-358 (1991).

69 Breneman CM, Thompson TR, Rhem M, Dung $M$. Electron density modeling of large systems using the transferable atom equivalent method. Comput. Chem. 19, 161-179 (1995).

70 Breneman CM, Rhem M. QSPR analysis of HPLC column capacity factors for a set of high-energy materials using electronic van der Waals' surface property descriptors computed by transferable atom equivalent method. J. Comput. Chem. 18, 182-197 (1997).

71 Sukumar N, Breneman CM. QTAIM in drug discovery and protein modeling. In: The Quantum Theory of Atoms in Molecules: from Solid State to DNA and Drug Design. Matta CF, Boyd RJ (Eds). Wiley-VCH, Weinheim, Germany 473-498 (2007).

72 Ramsey NF. Electron coupled interactions between nuclear spins in molecules. Phys. Rev. 91, 303-307 (1953).

73 Stephen MJ. A variational method for calculating nuclear spin-spin interactions in molecules. Proc. Roy. Soc. London A 243, 274-280 (1957).

74 Wilkens SJ, Westler WM, Markley JL, Weinhold F. Natural J-coupling analysis: interpretation of scalar J-couplings in terms of natural bond orbitals. J. Am. Chem. Soc. 123, 12026-12036 (2001).

75 Helgaker T, Jaszuński M, Ruud K. Ab initio methods for the calculation of NMR shielding and indirect spin-spin coupling constants. Chem. Rev. 99, 293-352 (1999).

76 Watson MA, Salek P, Macak P, Jaszuński M, Helgaker T. The calculation of indirect nuclear spin-spin coupling constants in large molecules. Chem. Eur. J. 10, 4627-4639 (2004).

77 Alkorta I, Elguero J. Review on DFT and ab initio calculations of scalar coupling constants. Int. J. Mol. Sci. 4, 64-92 (2003).

78 Matta CF, Hernández-Trujillo J, Bader RFW. Proton spin-spin coupling and electron delocalisation. J. Phys. Chem. A 106, 7369-7375 (2002). 
79 Matta CF. Applications of the quantum theory of atoms in molecules to chemical and biochemical problems. PhD thesis. McMaster University, Hamilton, Canada (2002).

80 N. Castillo, Matta CF, Boyd RJ. Fluorinefluorine spin-spin coupling constants: correlations with the delocalization index and with the internuclear separation. J. Chem. Inf. Mod. 45, 354-359 (2005).

81 Pople AJ, Schneider WG, Bernstein HJ. High-Resolution Nuclear Magnetic Resonance. McGraw-Hill Book Co. Inc, New York, USA (1959).

82 Tomasi J, Cappelli C, Mennucci B, Cammi R. From molecular electrostatic potentials to solvations models and ending with biomolecular photophysical processes. In: Quantum Biochemistry: Electronic Structure and Biological Activity (Volume 1). Matta CF (Ed.). Wiley-VCH, Weinheim, Germany 131-170 (2010).

83 Bonaccorsi R, Scrocco E, Tomasi J. Approximate expression of electrostatic molecular potential in terms of completely transferable group contributions. J. Am. Chem. Soc. 99, 4546-4554 (1977).

84 Bonaccorsi R, Scrocco E, Tomasi J. Group contributions to electrostatic molecular potential. J. Am. Chem. Soc. 98, 4049-4054 (1976).

85 Petrongolo C, Tomasi J. The use of electrostatic molecular potential in quantum pharmacology. 1. Ab initio results. Int. J. Quantum Chem. Quantum Biol. Symp. No. 2. 181-190 (1975).

86 Chemical Applications of Atomic and Molecular Electrostatic Potentials. Reactivity, Structure, Scattering, and Energetics of Organic, Inorganic, and Biological Systems. Politzer P, Truhlar DG (Eds). Plenum Press, New York, USA (1981).

87 Murray JS, Politzer P. Electrostatic potentials: chemical applications. In: Encyclopedia of Computational Chemistry. Schleyer PvR (Ed.) John Wiley \& Sons, Chichester, UK (1998).

88 Politzer P, Murray JS, Peralta-Inga Z. Molecular surface electrostatic potentials in relation to noncovalent interactions in biological systems. Int. J. Quantum Chem. 85, 676-684 (2001).

89 Gonzalez OG, Murray JS, Peralta-Inga Z, Politzer P. Computed molecular surface electrostatic potentials of two groups of reverse transcriptase inhibitors: relationships to anti-HIV-1 activities. Int. J. Quantum Chem. 83, 115-121 (2001).

90 Murray JS, Politzer P. The use of the molecular electrostatic potential in medicinal chemistry. In: Quantum Medicinal Chemistry. Carloni P, Alber F (Eds). Wiley-VCH, Weinheim, Germany 233-254 (2003).
91 Babu K, Ganesh V, Gadre SR, Ghermani NE. Tailoring approach for exploring electron densities and electrostatic potentials of molecular crystals. Theor. Chem. Acc. 111, 255 (2004).

92 Politzer P, Murray JS. Molecular electrostatic potentials and chemical reactivity. In: Reviews in Computational Chemistry. John Wiley \& Sons, Inc, Hoboken, NJ, USA (2007).

93 Herr R. 5-Substituted-1H-tetrazoles as carboxylic acid isosteres: medicinal chemistry and synthetic methods. J. Bioorg. Med. Chem. 10, 3379-3393 (2002).

94 Biot C, Bauer H, Schirmer RH, DavioudCharvet E. 5-Substituted tetrazoles as bioisosteres of carboxylic acids. Bioisosterism and mechanistic studies on glutathione reductase inhibitors as antimalarials. J. Med. Chem. 47, 5972-5983 (2004).

95 Yuan H, Silverman RB. New substrates and inhibitors of $\gamma$-aminobutyric acid aminotransferase containing bioisosteres of the carboxylic acid group: Design, synthesis, and biological activity. Bioorg. Med. Chem. 14, 1331-1338 (2006).

96 Yuan H, Silverman RB. Structural modifications of $(1 S, 3 S)$-3-amino-4-difluoro methylenecyclopentanecarboxylic acid, a potent irreversible inhibitor of GABA aminotransferase. Bioorg. Med. Chem. Lett. 17, 1651-1654 (2007).

97 Burgos-Lepley CE, Thompson LR, Kneen $\mathrm{CO}$ et al. Carboxylate bioisosteres of gabapentin. Bioorg. Med. Chem. Lett. 16, 2333-2336 (2006).

98 Kohn W, Sham LJ. Self consistent equations including exchange and correlation effects. Phys. Rev. A 140 (4A), 1133-1138 (1965).

99 Bader RFW, Jones GA. The electron density distributions in hydride molecules, I, the water molecule. Can. J. Chem. 41, 586-606 (1963).

100 Bader RFW, Jones GA. The electron density distributions in hydride molecules, III, the hydrogen fluoride molecule. Can. J. Chem. 41, 2251-2264 (1963).

101 Bader RFW, Jones GA. The electron density distribution in hydride molecules. The ammonia molecule. J. Chem. Phys. 38, 2791-2802 (1963).

102 Bader RFW. binding regions in polyatomic molecules and electron density distributions. J. Am. Chem. Soc. 86, 5070-5075 (1964).

103 Dovesi R, Saunders VR, Roetti. CRYSTAL98 (1998).

104 Becke A. A new mixing of Hartree-Fock and local density-functional theories. J. Chem. Phys. 98, 1372-1377 (1993).
105 Lee C, Yang W, Parr R. Development of the Colle-Salvetti correlation-energy formula into a functional of the electron-density. Phys. Rev. B 37, 785-789 (1988).

106 Matta CF. Quantum Biochemistry: Electronic Structure and Biological Activity (Volumes 1 \& 2). Wiley-VCH, Weinheim, Germany (2010).

107 Carloni P, Alber FE (Eds). Quantum Medicinal Chemistry. Wiley-VCH, Weinheim, Germany (2003).

108 Brändas EJ, Kryachko ES (Eds). Fundamental World of Quantum Chemistry: A Tribute to the Memory of Per-Olov Löwdin. Kluwer Academic Publishers, Dordrecht, The Netherlands (2003).

109 Becker OM. MacKerell AD Jr, Roux B, Watanabe M. Computational Biochemistry and Biophysics. Marcel Dekker Inc, NY, USA (2001).

110 Eriksson LA (Eds). Theoretical Biochemistry - Processes and Properties of Biological Systems. Elsevier Science BV, Amsterdam, The Netherlands (2001).

111 Greenberg A, Breneman C, Liebman JFE. The Amide Linkage: Selected Structural Aspects in Chemistry, Biochemistry, and Materials Science. John Wiley and Sons, Inc, New York, USA (2000).

112 Sapse AM. Molecular Orbital Calculations for Amino Acids and Peptides. Birkhäuser, Boston, USA (2000).

113 Biegler-König FW, Bader RFW, T.-Tang H. Calculation of the average properties of atoms in molecules. II. J. Comput. Chem. 13, 317-328 (1982).

114 Popelier PLA. MORPHY, a program for an automatic atoms in molecules analysis. Comp. Phys. Comm. 1996, 93, 212-240.

115 Popelier PLA. MORPHY, Manchester University, England, UK (1998).

116 Biegler-König FW. Calculation of atomic integration data. J. Comput. Chem. 21, 1040-1048 (2000).

117 Biegler-König FW, Schönbohm J, Bayles D. AIM2000 - a program to analyze and visualize atoms in molecules. J. Comput. Chem. 22, 545-559 (2001).

118 Keith TA. AIMAll (2009).

119 Gatti C. TOPOND, CNR-CSRSRC. Milano, Italy (1998).

120 Espinosa E, Molins E, Lecomte C. Hydrogen bond strengths related by topological analyses of experimentally observed electron densities. Chem. Phys. Lett. 285, 170-173 (1998).

121 Housset D, Benabicha F, Pichon-Pesme $\mathrm{V}$ et al. Towards the charge-density study of proteins: A room-temperature scorpion-toxin 
structure at $0.96 \AA$ resolution as a first test case. Acta Cryst. D56, 151-160 (2000).

122 Benabicha F, Pichon-Pesme V, Jelsch C, Lecomte C, Khmou A. Experimental charge density and electrostatic potential of glycyl-L-threonine dihydrate. Acta Cryst. B56, 155-165 (2000).

123 Leherte L, Guillot B, Vercauteren DP et al. Topological analysis of proteins as derived from medium and high-resolution electron density: applications to electrostatic properties. In: The Quantum Theory of Atoms in Molecules: From Solid State to DNA and Drug Design. Matta CF, Boyd RJ (Eds). Wiley-VCH, Weinheim, Germany 285-315 (2007).

124 Dittrich B, Koritsánszky T, Grosche M et al. Reproducability and transferability of topological properties. experimental charge density of the hexapeptide cyclo-(D, L-Pro)2-(L-Ala) 4 monohydrate. Acta Cryst. B58, 721-727 (2002).

125 Scheins S, Messerschmidt M, Luger P. Submolecular partitioning of morphine hydrate based on its experimental charge density at 25 K. Acta Cryst. B61, 443-448 (2005).

126 P. Luger, Dittrich B. Fragment transferability studied theoretically and experimentally with QTAIM - implications for electron density and invariom modeling. In: The Quantum Theory of Atoms in Molecules: From Solid State to DNA and Drug Design. Matta CF, Boyd RJ (Eds). Wiley-VCH, Weinheim, Germany 317-341 (2007).

127 Luger P. Fast electron density methods in the life sciences - a routine application in the future? Org. Biomolec. Chem. 5, 2529-2540 (2007).

128 Koritsanszky TS, Howard S, Macchi P et al. XD Program. Free University of Berlin, Germany. University of Wales, Cardiff, UK. Universita di Milano, UK. CNR-ISTM, Milano, UK. University of Glasgow UK. State University of New York, Buffalo, USA. University of Nancy, France (2003).

129 B. Guillot, Viry L, Guillot R, Lecomte C, Jelsch CJ. Refinement of proteins at subatomic resolution with MoPro. J. App. Cryst. 34, 214-223 (2001).

130 Jelsch C, Guillot B, Lagoutte L, Lecomte C. Advances in proteins and small molecules. charge density refinement methods using software MoPro. J. App. Cryst. 38, 38-54 (2005).
131 Wiberg KB, Bader RFW, Lau CDH. Theoretical analysis of hydrocarbons properties. 2. Additivity of group properties and the origin of strain energy. J. Am. Chem. Soc. 109, 1001-1012 (1987).

132 Keith TA, Bader RFW. Calculation of magnetic response properties using atoms in molecules. Chem. Phys. Lett. 194, 1-8 (1992).

133 Keith TA, Bader RFW. Use of electron charge and current distributions in the determination of atomic contributions to magnetic properties. Int. J. Quantum Chem. 60, 373-379 (1996).

134 Bader RFW, Keith TA, Gough KM, Laidig KE. Properties of atoms in molecules: additivity and transferability of group polarizabilities. Mol. Phys. 75, 1167-1189 (1992).

135 Gough KM, Yacowar MM, Cleve RH, Dwyer RJ. Analysis of molecular polarizabilities and polarizability derivatives in $\mathrm{H}_{2}, \mathrm{~N}_{2}, \mathrm{~F}_{2}, \mathrm{CO}$, and $\mathrm{HF}$, with the theory of atoms in molecules. Can. J. Chem. 74, 1139-1144 (1996).

136 Gough KM, Srivastava HK, Belohorcová K. Molecular polarizability and polarizability derivatives in cyclohexane analyzed with the theory of atoms in molecules. J. Phys. Chem. 98, 771-776 (1994).

137 Gough KM, Srivastava HK. Electronic charge flow and Raman trace scattering intensities for $\mathrm{CH}$ stretching vibrations in $n$-pentane. J. Phys. Chem. 100, 5210-5216 (1996).

138 Haiduke RLA, Bruns RE. An atomic charge-charge flux-dipole flux atom-inmolecule decomposition for molecular dipole-moment derivatives and infrared fundamental intensities. J. Phys. Chem. A 109, 2680-2688 (2005).

139 da Silva JV, Haiduke RLA, Bruns RE. QTAIM charge-charge flux-dipole flux models for the infrared fundamental intensities of the fluorochloromethanes. J. Phys. Chem. A 110, 4839-4845 (2006).

140 Austen MA. A new procedure for determining bond orders in polar molecules, with applications to phosphorus and nitrogen containing systems. PhD thesis. McMaster University, Hamilton, Canada (2003).

141 Wang YG, Matta CF, Werstiuk NH. Comparison of localization and delocalization indices obtained with Hartree-Fock and conventional correlated methods: effect of Coulomb correlation. J. Comput. Chem. 24, 1720-1729 (2003).
142 Bohórquez HJ, Obregón M, Cárdenas C et al. Electronic energy and multipolar moments characterize amino acid side chains into chemically related groups. J. Phys. Chem. A. 107, 10090-10097 (2003).

143 Bohorquez HJ, Cardenas C, Matta CF, Boyd RJ, Patarroyo ME. Methods in biocomputational chemistry: a lesson from the amino acids. In: Quantum Biochemistry: Electronic Structure and Biological Activity. Matta CF, Boyd RJ (Eds). Wiley-VCH, Weinheim, Germany 403-421 (2010).

144 Matta CF, Bader RFW. An atoms-inmolecules study of the genetically-encoded amino acids. I. Effects of conformation and of tautomerization on geometric, atomic, and bond properties. Proteins: Struct. Funct. Genet. 40, 310-329 (2000).

145 Matta CF, Bader RFW. Atoms-in-molecules study of the genetically-encoded amino acids. II. Computational study of molecular geometries. Proteins: Struct. Funct. Genet. 48, 519-538 (2002).

146 Song M, Breneman CM, Bi J et al. Prediction of protein retention times in anion-exchange chromatography systems using support vector regression. J. Chem. Inf. Comput. Sci. 42, 1347-1357 (2002).

147 Popelier PLA, Aicken FM. Atomic properties of amino acids: computed atom types as a guide for future force-field design. Chemphyschem 4, 824-829 (2003).

148 Popelier PLA, Aicken FM. Atomic properties of selected biomolecules: quantum topological atom types of carbon occurring in natural amino acids and derived molecules. J. Am. Chem. Soc. 125, 12841292 (2003).

149 Matta CF. Theoretical reconstruction of the electron density of large molecules from fragments determined as proper open quantum systems: the properties of the oripavine PEO, enkephalins, and morphine. J. Phys. Chem. A 105, 11088-11101 (2001).

\section{Websites}

201 Aimpac. Download page. www.chemistry.mcmaster.ca/aimpac

202 AIM2000 program. Download page. http://gauss.fh-bielefeld.de/aim2000 